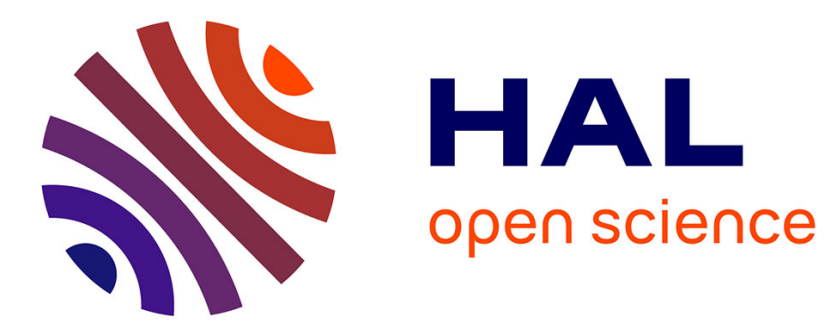

\title{
Interpretation and generalization of complexity pursuit for the blind separation of modal contributions
}

Jérôme Antoni, Roberto Castiglione, Luigi Garibaldi

\section{To cite this version:}

Jérôme Antoni, Roberto Castiglione, Luigi Garibaldi. Interpretation and generalization of complexity pursuit for the blind separation of modal contributions. Mechanical Systems and Signal Processing, 2017, 85, pp.773 - 788. 10.1016/j.ymssp.2016.09.009 . hal-01714958

\section{HAL Id: hal-01714958 \\ https://hal.science/hal-01714958}

Submitted on 3 Sep 2021

HAL is a multi-disciplinary open access archive for the deposit and dissemination of scientific research documents, whether they are published or not. The documents may come from teaching and research institutions in France or abroad, or from public or private research centers.
L'archive ouverte pluridisciplinaire HAL, est destinée au dépôt et à la diffusion de documents scientifiques de niveau recherche, publiés ou non, émanant des établissements d'enseignement et de recherche français ou étrangers, des laboratoires publics ou privés. 


\title{
Interpretation and generalization of complexity pursuit for the blind separation of modal contributions
}

\author{
Jerome Antoni $^{\mathrm{a}}$, Roberto Castiglione ${ }^{\mathrm{a}, \mathrm{b}, *}$, Luigi Garibaldi $^{\mathrm{b}}$ \\ a Laboratoire Vibrations Acoustique, University of Lyon, INSA-Lyon, Lyon F-69621, France \\ b Dipartimento di Ingegneria Meccanica e Aerospaziale, Politecnico di Torino, Corso Duca degli Abruzzi 24, 10129 Torino, Italy
}

\section{A R T I C L E I N F O}

\section{Keywords:}

Operational modal analysis

Blind source separation

System identification

Complexity pursuit

SOBI algorithm

\begin{abstract}
A B S T R A C T
Complexity pursuit (CP) has recently been proposed as an elegant and simple solution to blindly (i.e. without measuring the inputs) separate the modal contributions in the vibration responses of a structure. This potentially finds considerable interest in operational modal analysis and related applications. This paper analyses the theoretical ins and outs of the method. It also revises its physical interpretation in the modal analysis context. $\mathrm{CP}$ is found to separate components which are the least dispersive (i.e. invariant under linear filtering), a property that well characterizes the modal responses of lightly damped systems. However, it is also found to suffer from the same limitations as other blind source separation methods used in the state-ofthe-art, namely the difficulty to separate strongly coupled modes and to identify complex mode shapes. Finally a generalization of $\mathrm{CP}$ is proposed which intends to widen its applicability. Interestingly, the generalized CreP happens to include the well-known SOBI algorithm as a particular case.
\end{abstract}

\section{Introduction}

Complexity Pursuit (CP), a new blind source separation (BSS) technique, was recently introduced in [1-3] and demonstrated to decompose the vibration responses of a structure into individual modal contributions. Such a technique is of particular interest within the context of operational modal analysis (OMA) due to its ability to blindly (i.e. without measuring the inputs) decouple a multiple-degree-of-freedom system into a set of single-degree-of-freedom components, as demonstrated in precursory works [58,10-13] and in later developments [14]. In particular, it can greatly simplify the subsequent identification task required for extracting the modal information from the system responses: the global modal parameters (natural frequencies and damping ratios) can easily be identified by using single-degree-of-freedom methods and the mode shape estimated from the inverse of the separation matrix. The BSS technique of [1] is an adaptation of the complexity pursuit (CP) principle initially formulated in [4] in a statistical learning context (note that [15] independently formulated a BSS method based on a similar idea). Basically, it consists in finding a modal filter intended to extracting an individual modal contribution by minimizing the energy ratio between two filtered versions of the output signals. Namely, using the notations of [1], let the $n \times 1$ column vector

$$
\mathbf{x}(t)=\boldsymbol{\Phi} \mathbf{q}(t)=\sum_{i=1}^{n} \boldsymbol{\varphi}_{i} q_{i}(t)
$$

\footnotetext{
Abbreviations: AMUSE, Algorithm for multiple unknown signals extraction (algorithm); BSS, Blind source separation; CP, Complexity Pursuit (algorithm); DOF, Degrees of freedom; GCP, Generalized Complexity Pursuit (algorithm); OMA, Operational modal analysis; SOBI, Second order blind identification (algorithm)

* Corresponding author at: Laboratoire Vibrations Acoustique, University of Lyon, INSA-Lyon, Lyon F-69621, France.

E-mail addresses: jerome.antoni@insa-lyon.fr (J. Antoni), roberto.castiglione@polito.it (R. Castiglione), luigi.garibaldi@polito.it (L. Garibaldi).
} 
denotes the observed vibration responses of a structure at time $t$ produced by a mixture of modal coordinates $q_{i}(t)$ weighted by mode shapes $\boldsymbol{\varphi}_{i}, i=1, \ldots, n$ (columns of the $n \times n$ modal matrix $\boldsymbol{\Phi}$ ). The objective is to find a modal filter $\mathbf{w}_{i}($ a $1 \times n$ raw vector) such that

$$
y_{i}(t)=\mathbf{w}_{i} \mathbf{x}(t)
$$

returns an estimate of $q_{i}(t)$ up to a scaling factor, from which global modal parameters can be subsequently recovered, on the one hand. One the other hand, the inverse of matrix $\mathbf{W}$ made of the rows $\mathbf{w}_{i}, i=1, \ldots, n$ is an estimate of the modal matrix $\boldsymbol{\Phi}$ that contains information on the mode shapes. The principle of CP is to estimate $\mathbf{w}_{i}$ such as to minimize the ratio

$$
\rho_{i}=\frac{\left\langle\left. y_{i}^{(1)}(t)\right|^{2}\right\rangle}{\left\langle\left. y_{i}^{(2)}(t)\right|^{2}\right\rangle}=\frac{\mathbf{w}_{i}\left(\mathbf{x}^{(1)}(t) \mathbf{x}^{(1)}(t)^{T}\right) \mathbf{w}_{i}^{T}}{\mathbf{w}_{i}\left(\mathbf{x}^{(2)}(t) \mathbf{x}^{(2)}(t)^{T}\right) \mathbf{w}_{i}^{T}}
$$

where $\langle\cdots\rangle$ stands for the time average operator and superscript $k=1,2$ symbolically represents two different filtered versions of the same signals. In its original formulation, $\mathbf{x}^{(1)}(t)$ (resp. $\left.\mathbf{x}^{(2)}(t)\right)$ is the residual between the actual signals $\mathbf{x}(t)$ and a long-term predictor $\overline{\mathbf{x}}^{(1)}(t)$ (resp. short -term predictor $\overline{\mathbf{x}}^{(2)}(t)$ )

$$
\begin{aligned}
& \left\{\begin{array}{l}
\mathbf{x}^{(k)}(t)=\overline{\mathbf{x}}^{(k)}(t)-\mathbf{x}(t) \\
\overline{\mathbf{x}}^{(k)}(t)=\lambda_{k} \overline{\mathbf{x}}^{(k)}(t-1)+\left(1-\lambda_{k}\right) \mathbf{x}(t-1)
\end{array}\right. \\
& 0 \leq \lambda_{1}<\lambda_{2} \leq 1, \quad k=1,2
\end{aligned}
$$

[1-3] used $\lambda_{1}=2^{-1 / 900000}$ and $\lambda_{2}=0.5$ by default.

As stated in [1], the rationale beyond criterion (3) is to seek a separation vector $\mathbf{w}_{i}$ that yields the "least complexity and thus approaches the (simplest) source signal, where the complexity is robustly measured by temporal predictability". Using the authors' words, CP is "computational efficient, user-friendly, and automatic, requiring little expertise interactions for implementations". Indeed, the minimization of criterion (3) with respect to $\mathbf{w}_{i}$ simply amounts to computing the generalized eigenvectors of the crosscorrelation matrices $\left\langle\mathbf{x}^{(1)}(t) \mathbf{x}^{(1)}(t)^{T}\right\rangle$ and $\left\langle\mathbf{x}^{(2)}(t) \mathbf{x}^{(2)}(t)^{T}\right\rangle$ (see Section 3.1).

A first objective of this paper is to provide the theoretical foundation and physical interpretation of the CP that are lacking in [1]. In view of its potential importance to OMA, it is imperative to establish at the onset whether it is capable of separating modal contributions in the general scenario and, if not, to delineate its limits. In particular, the question arises as whether it can resolve highly coupled modes (e.g. closely spaced frequencies and/or strongly damped modes) and complex mode shapes, two configurations which are still challenging to cutting-edge BSS techniques [14]. Second, it is also compulsory to compare its performance against SOBI [17], a state-of-the-art BSS technique used in OMA which has been standing as a point of reference since a few years $[7,8,10,14]$.

A second objective of the paper is to propose a generalization of CP that is shown to apply more widely and provides perspective for the proposal of new BSS algorithms.

The main results of this paper are summarized hereafter:

1) The solutions of CP, as formulated by Eqs. (2)-(4), are pure sines. Strictly speaking, this generally precludes the exact recovery of vibration modes as soon as damping is present in the system.

2) However, very good separation of lightly damped modes is to be expected provided the built-in filters of CP are smooth enough to be considered as approximately constant across the mode bandwidths.

3) The original formulation of CP in terms of short and long-term predictors (Eq. (4)) can be generalized to the consideration of any type of filters, provided they are smooth enough in the sense of point (2). This makes obsolete the interpretation of CP as seeking for the least complex components that are "maximally predictable".

4) The physical interpretation of the $\mathrm{CP}$ is to seek vibration components that remain invariant under arbitrary (linear) filtering. In terms of waveforms, these are components which are as least dispersive as possible, that is nearly invariant under linear filtering. Non-dispersion is an intrinsic property of pure sines, yet it can be approached remarkably well by lightly damped modes whose modal coordinates resemble slowly modulated sinusoids. Least complexity in CP is therefore to be measured by "dispersion" rather than by "predictability".

5) $\mathrm{CP}$ is generally unable to recover complex mode shapes (e.g. in the case of non-proportional damping) since the generalized eigenvectors of square real symmetric matrices $\left\langle\mathbf{x}^{(1)}(t) \mathbf{x}^{(1)}(t)^{T}\right\rangle$ and $\left\langle\mathbf{x}^{(2)}(t) \mathbf{x}^{(2)}(t)^{T}\right\rangle$ are real-valued, unless specific pre-processing is used as suggested in [10].

6) CP presents a strong analogy with AMUSE, the two time-lag version of SOBI [18]. Some particular choices of the built-in filters can make it identical to AMUSE.

7) Simulations show that CP is not superior to SOBI in the general case and that it suffers from the same difficulty to separate strongly coupled modes and complex mode shapes.

8) A generalization of the original CP algorithm is proposed that involves an arbitrary number of filters. This involves an approximate joint diagonalization of a set of cross-correlation matrices which is likely to improve the performance of the plain-vanilla CP method. The generalization includes SOBI as a particular case.

9) Several sets of filters are tested on simulated and real data in order to demonstrate how to optimize the separation of vibration components. One advantage of the generalized CP is to provide a versatile algorithm that is intended to shortcut this step. 
The proofs of these results are given in the rest of the paper. Section 2 first establishes the optimality condition under which criterion (3) is minimized. Next, Section 3 investigates the behavior of the criterion when trying to separate modes of a dissipative (i. e. non-zero damping) system and discusses the design of optimal filters. Section 4 then introduces the generalized version of CP. Finally, Section 5 provides some comparisons of CP, its generalized version, and SOBI by means of numerical experiments, Section 6 experimentally addresses the optimization of filters, and Section 7 demonstrates the applicability of the generalized algorithm on real data.

\section{Optimality condition}

The very first matter to investigate is which type of signals is a solution of the minimum of criterion (3). In order to do so, let us use Parseval's identity in Eq. (3),

$$
\rho_{i}=\frac{\int_{0}^{\pi}\left|Y_{i}^{(1)}(\omega)\right|^{2} d \omega}{\int_{0}^{\pi}\left|Y_{i}^{(2)}(\omega)\right|^{2} d \omega}=\frac{\int_{0}^{\pi}\left|H_{1}(\omega) Y_{i}(\omega)\right|^{2} d \omega}{\int_{0}^{\pi}\left|H_{2}(\omega) Y_{i}(\omega)\right|^{2} d \omega}=\frac{U}{V}
$$

where $Y_{i}^{(k)}(\omega)=H_{k}(\omega) Y_{i}(\omega), k=1,2$ stands for the Fourier transform of $y_{i}^{(k)}(t)$ and $H_{k}(\omega)$ for the transfer function that relates the filtered signal $y_{i}^{(k)}(t)$ to its original version $y_{i}(t)$. For instance, according to Eq. $(4), H_{k}(\omega)=\lambda_{k}\left(e^{-j \omega}-1\right) /\left(1-\lambda_{k} e^{-j \omega}\right), j^{2}=-1$. Following the principles of variational calculus, let us assume that $Y_{i}(\omega)$ is close to its optimal solution $Y_{i}^{o}(\omega)$, that is $Y_{i}(\omega)=Y_{i}^{o}(\omega)+\delta Y_{i}(\omega)$ where $\delta Y_{i}(\omega)$ stands for a small perturbation. Thus

$$
\delta \rho_{i}=\rho_{i}\left(Y_{i}(\omega)\right)-\rho_{i}\left(Y_{i}^{\omega}(\omega)\right) \approx \frac{\int_{0}^{\pi} Y_{i}^{\rho}(\omega)^{*}\left(V\left|H_{1}(\omega)\right|^{2}-U\left|H_{2}(\omega)\right|^{2}\right) \delta Y_{i}(\omega) d \omega}{V^{2}}
$$

where symbol * denotes the conjugate operator, constants $U$ and $V$ where defined in Eq. (5), and higher-order terms in $\delta Y_{i}(\omega)$ have been neglected (see proof in Appendix A). The optimality condition requires that $\delta \rho_{i}=0$ whatever the perturbation $\delta Y_{i}(\omega)$, which implies that the actual solution $Y_{i}^{o}(\omega)$ must verify the equation

$$
Y_{i}^{\omega}(\omega)^{*}\left(V\left|H_{1}(\omega)\right|^{2}-U\left|H_{2}(\omega)\right|^{2}\right)=0 .
$$

Ignoring the trivial solution $Y_{i}^{o}(\omega)=0$, one must have $U\left|H_{2}(\omega)\right|^{2}=V\left|H_{1}(\omega)\right|^{2}$. But this cannot hold identically, for all frequencies, otherwise ratio $\rho_{i}$ would be invariant whatever the value of $Y_{i}^{o}(\omega)$. Thus, the only putative solution is in the form $Y_{i}^{o}(\omega)=\sum_{p} A_{p} \delta\left(\omega-\omega_{p}\right)$ where $\left\{\omega_{p}\right\}$ is a finite set of fixed frequencies. After insertion into Eq. (7), this implies

$$
\frac{U}{V}=\frac{\left|H_{1}\left(\omega_{p}\right)\right|^{2}}{\left|H_{2}\left(\omega_{p}\right)\right|^{2}} \text { for all } p
$$

At the same time such a solution yields

$$
\rho_{2}=\frac{U}{V}=\frac{\int_{0}^{\pi}\left|H_{1}(\omega) Y_{i}^{o}(\omega)\right|^{2} d \omega}{\int_{0}^{\pi}\left|H_{2}(\omega) Y_{\mathrm{i}}^{\rho}(\omega)\right|^{2} d \omega}=\frac{\sum_{p} A_{p}\left|H_{1}\left(\omega_{p}\right)\right|^{2}}{\sum_{p} A_{p}\left|H_{2}\left(\omega_{p}\right)\right|^{2}} .
$$

Upon identification of Eqs. (8) and (9), one must have

$$
\frac{\sum_{p} A_{p}\left|H_{1}\left(\omega_{p}\right)\right|^{2}}{\sum_{p} A_{p}\left|H_{2}\left(\omega_{p}\right)\right|^{2}}=\frac{\left|H_{1}\left(\omega_{p}\right)\right|^{2}}{\left|H_{2}\left(\omega_{p}\right)\right|^{2}} \forall p
$$

which cannot be satisfied in general for arbitrary transfer functions (e.g. arbitrary values of $\lambda_{1}$ and $\lambda_{2}$ in Eq. (4)) unless all $A_{p}$ 's are nil but one. Therefore the pure sine $Y_{i}^{o}(\omega)=A_{o} \delta\left(\omega-\omega_{o}\right)$ (with arbitrary amplitude $A_{o}$ and frequency $\left.\omega_{o}\right)$ is the minimizer of the CP criterion, a result which is consistent with other BSS criteria recently proposed in the field of modal analysis [19].

It is emphasized that this result has been established without any statistical assumption on the modal contributions. In particular, they have not been assumed independent as is the current practice in BSS. Indeed, there is no physical reason why independence should hold in modal analysis in general [14], the reason why it is not taken as a premise in this work.

Another remarkable implication of the optimality condition (7) is that it applies independently of a particular form of the transfer functions, $H_{1}(\omega)$ and $H_{2}(\omega)$, as long as they are not identical. Indeed, the conclusion that a pure sine is the only solution to the CP principle has been arrived at without using the structure given in Eq. (4). Alternatively, assuming such a structure would have not changed the conclusion. This makes obsolete the interpretation of CP as seeking for least complex components that are maximally predictable, as given in [1-4]. Rather, complexity should be measured by the propensity of a component to be dispersive. This also paves the way to the design of more efficient BSS algorithms based on different and optimized filters (see Section 4).

\section{Identifiability of damped modes}

The optimality condition found in the previous section states that if a sinusoid is present in a set of vibration responses, then it 
will be extracted by CP. Obviously, such a situation is quite unrealistic in OMA where the generation of pure sines would require the system to be perfectly conservative (i.e. without damping). It is therefore compulsory to investigate the capability of $\mathrm{CP}$ to (approximately) separate damped modes in a real-world scenario.

\subsection{Inherent working assumptions of $C P$}

The results of this subsection are established under the assumption of a steady state and stationary regime (they equally hold for transient responses ${ }^{1}$ ). Without loss of generality, let us express the modal contributions of a dissipative structure as slowly modulated sinusoids. Thus, the overall vibration responses read

$$
\mathbf{x}(t)=\boldsymbol{\Phi} \mathbf{q}(t)=\sum_{i=1}^{n} \boldsymbol{\varphi}_{i} A_{i}(t) \cos \left(\omega_{i} t+\phi_{i}(t)\right) .
$$

where $A_{i}(t)$ and $\phi_{i}(t)$ are slow amplitude and phase modulation functions, respectively. Note that at this stage no additive noise is assumed for the sake of simplicity; robustness of $\mathrm{CP}$ with respect to additive noise will be experimentally investigated in subsection 5.4. The next step is to filter these responses through transfer functions $H_{k}(\omega), k=1,2$. Provided that $H_{k}(\omega)$ is smooth enough so that it can be assumed nearly constant across the spectrum of $A_{i}(t)$ and $\phi_{i}(t)$, then

$$
\mathbf{x}_{k}(t) \approx \sum_{i} \boldsymbol{\varphi}_{i} A_{i}(t)\left|H_{k}\left(\omega_{i}\right)\right| \cos \left(\omega_{i} t+\phi_{i}(t)+\angle H_{k}\left(\omega_{i}\right)\right), \quad k=1,2
$$

holds to a very good approximation, where $\angle H_{k}\left(\omega_{i}\right)$ denotes the phase of the transfer function such that $H_{k}=\left|H_{k}\right| \exp \left(j \angle H_{k}\right)$. The last step is to compute the cross-correlation matrices of the filtered signals, $\left\langle\mathbf{x}_{k}(t) \mathbf{x}_{k}(t)^{T}\right\rangle, k=1,2$, as required in the CP criterion (3). This involves cross-correlations

$$
\left\langle A_{i}(t) A_{j}(t) \cos \left(\omega_{i} t+\phi_{i}(t)+\angle H_{k}\left(\omega_{i}\right)\right) \cos \left(\omega_{j} t+\phi_{j}(t)+\angle H_{k}\left(\omega_{j}\right)\right)\right\rangle
$$

which are nearly zero provided that the distance between resonance frequencies $\omega_{i}$ and $\omega_{j}$ is greater than the spectral bandwidth of $A_{i}(t)$ and $\phi_{i}(t)$. Therefore, the cross-correlation matrix reads

$$
\left(\mathbf{x}^{(k)}(t) \mathbf{x}^{(k)}(t)^{T}\right) \approx \Phi \mathbf{D}_{k} \Phi^{T}
$$

with $\boldsymbol{\Phi}$ the modal matrix given in Eq. (1) and $\mathbf{D}_{k}$ a diagonal matrix whose $i$-th element is returned by

$$
\left[\mathbf{D}_{k}\right]_{i} \approx \frac{1}{2} \bar{A}_{i}^{2}\left|H_{k}\left(\omega_{i}\right)\right|^{2} .
$$

with $\bar{A}_{i}^{2}=\left\langle\left|A_{i}(t)\right|^{2}\right\rangle$. Finally, the CP criterion reads

$$
\rho_{i}=\frac{\mathbf{w}_{l} \Phi \mathbf{D}_{1} \Phi^{T} \mathbf{w}_{i}^{T}}{\mathbf{w}_{i} \boldsymbol{\Phi} \mathbf{D}_{2} \Phi^{T} \mathbf{w}_{l}^{T}}
$$

which is recognized as a Raleigh quotient, the minimum of which is returned by the smallest eigenvalue in the generalized eigenvalue decomposition $\boldsymbol{\Phi} \mathbf{D}_{1} \boldsymbol{\Phi}^{T} \mathbf{w}_{i}^{T}=\rho_{i} \boldsymbol{\Phi} \mathbf{D}_{2} \boldsymbol{\Phi}^{T} \mathbf{w}_{i}^{T}$ [16]. Repeating the procedure for all components $i=1, \ldots, n$ (the first component achieves the smallest minimum, the second one the next minimum and so on...), one obtains the matrix of generalized eigenvectors, $\mathbf{W}=\left[\mathbf{w}_{1}{ }^{T}, \ldots, \mathbf{w}_{n}^{T}\right]^{T}$ which, as well-known, makes $\mathbf{W} \boldsymbol{\Phi} \mathbf{D}_{1} \boldsymbol{\Phi}^{T} \mathbf{W}^{T}$ diagonal and $\mathbf{W} \boldsymbol{\Phi} \mathbf{D}_{2} \boldsymbol{\Phi}^{T} \mathbf{W}^{T}=\mathbf{I}$, the identity matrix (note in passing that these steps provide a more efficient algorithm to find the separation matrix of CP than the gradient ascent method initially proposed in [4]). Since $\mathbf{D}_{1}$ and $\mathbf{D}_{2}$ were found diagonal, this necessarily implies that

$$
\mathbf{W}=\mathbf{D}_{2}^{-\frac{1}{2}} \boldsymbol{\Phi}^{-1}=\operatorname{Diag}\left(\frac{1}{\sqrt{\frac{1}{2} \bar{A}_{i}^{2}} 1 H_{2}\left(\omega_{i}\right) \mid}\right) \boldsymbol{\Phi}^{-1},
$$

where Diag $\left(d_{i}\right)$ stands for the diagonal matrix with $i$-th element $d_{i}$. According to Eqs. (2) and (11), the separating matrix given by Eq. (17) correctly returns an estimate

$$
y_{i}(t)=w_{i} \mathbf{x}(t) \approx \frac{A_{i}(t)}{\sqrt{\frac{1}{2} \bar{A}_{i}^{2}}\left|H_{2}\left(\omega_{i}\right)\right|} \cos \left(\omega_{i} t+\phi_{i}(t)\right)
$$

of the $i$-th modal coordinate up to an unknown scaling factor and up to the effect of additive noise. In passing, the inverse matrix $\mathbf{W}^{-1}$ also returns an (unscaled) estimate of the modal matrix $\boldsymbol{\Phi}$.

So far, it seems reassuring that damped modes can indeed be separated by CP to a certain degree of approximation, even though they are not pure sines. The three assumptions which made this approximation valid are resumed hereafter:

\footnotetext{
${ }^{1}$ The proofs are essentially the same after redefining all time averages by finite-length summations.
} 


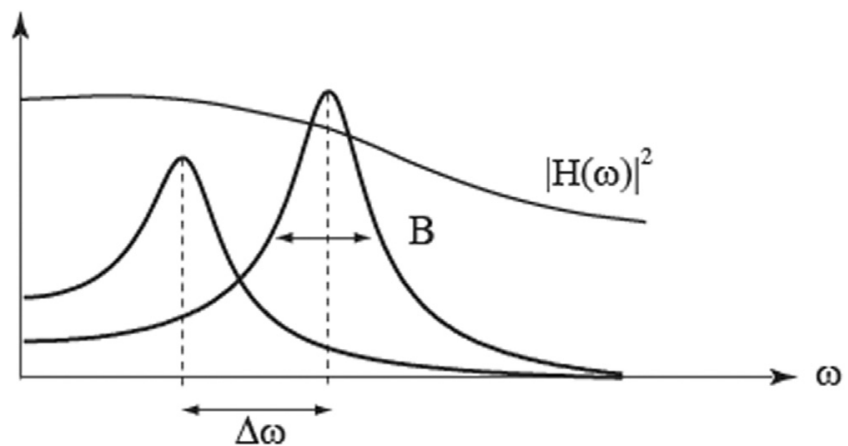

Fig. 1. Definition of the spectral separation $\Delta \omega$ and mode bandwidth $B$ used in conditions (19) and (20).

1. The effect of additive measurement noise can be neglected at the resonance frequencies (an assumption that is common to most BSS methods)

2. The frequency gains used in CP should be smooth enough to be nearly constant across the mode bandwidth $B$ (e.g. as defined by the half-power width). In other words, the effective impulse response length $\tau_{H}$ of the filters should be much shorter than the relaxation time $1 / B$ of the system:

$$
1 \gg \tau_{H} B \text {. }
$$

3. At the same time, the spectral separation $\Delta \omega$ of adjacent modes should be large enough so that they can be assumed nearly uncoupled, which may be safely stated as having the modal overlap factor $\mu(\omega)$ smaller than one

$$
1>\mu(\omega)=\frac{B}{\Delta \omega},
$$

Under these assumptions, damped modes will actually mimic the behavior of the ideal pure sines that are minimizers of the CP criterion - see Fig. 1. Any departure to conditions (19) and (20) will jeopardize the separation capability of CP. Alternatively, the degree to which conditions (19) and (20) are satisfied directly reflects the approximation error made in using CP when trying to separate damped modes.

Finally, a last necessary condition relates to the ability of CP to separate all damped modes active in a system. It requires that matrices $\boldsymbol{\Phi D}_{1} \boldsymbol{\Phi}^{T}$ and $\boldsymbol{\Phi D}_{2} \boldsymbol{\Phi}^{T}$ have distinct generalized eigenvalues

$$
\rho_{i} \approx \frac{\left|H_{1}\left(\omega_{i}\right)\right|^{2}}{\left|H_{2}\left(\omega_{i}\right)\right|^{\mid}}, \quad i=1, \ldots, n \text {. }
$$

\subsection{Revising the interpretation of $C P$}

The above results now make it clear that $\mathrm{CP}$ tries to separate components that are least invariant under arbitrary but smooth linear filters. Strictly speaking, pure sines are the only waveforms which are exactly non-dispersive under linear filtering. However, the property has been shown to hold approximately for damped modes provided dissipation is light enough in the sense of condition (19). In other words, the least complex components extracted by CP are the least dispersive possible. This suggests that the concept on "complexity" in CP should rather be measured by the propensity to dispersion rather than by predictability as originally formulated in [4].

\subsection{Connection with AMUSE}

When generalized to an arbitrary pair of linear filters, it appears the CP includes AMUSE [18] as a particular case, an algorithm which bears some historical reputation in BSS [9]. The connection is all the more important as it also suggests a link with SOBI [17] - to be revealed in Section 4 - the evolution of AMUSE which currently stands as a point of reference in BSS.

Briefly stated, the principle of AMUSE is to seek that separation matrix $\mathbf{W}$ which jointly diagonalizes a set of cross-correlation matrices $\left\langle\mathbf{x}(t) \mathbf{x}\left(t-\tau_{i}\right)^{T}\right\rangle, i=1,2$, at two time-lags $\tau_{1}$ and $\tau_{2}$. Specifically, it returns the rows $\mathbf{w}_{i} i=1, \ldots, n$ of matrix $\mathbf{W}$ that minimize

$$
\rho_{i}^{\text {AMUSE }}=\frac{\mathbf{w}_{i}\left\langle\mathbf{x}(t) \mathbf{x}\left(t-\tau_{1}\right)^{T}\right\rangle \mathbf{w}_{i}^{T}}{\mathbf{w}_{i}\left(\mathbf{x}(t) \mathbf{x}\left(t-\tau_{2}\right)^{T}\right\rangle \mathbf{w}_{i}^{T}}=\frac{\int_{0}^{\pi}\left|Y_{i}(\omega)\right|^{2} \cos \left(\omega \tau_{1}\right) d \omega}{\int_{0}^{\pi}\left|Y_{i}(\omega)\right|^{2} \cos \left(\omega \tau_{2}\right) d \omega}, \quad \pi_{1} \neq \tau_{2} .
$$

The resemblance with the CP criterion (5) is striking, where $\cos \left(\omega \tau_{1}\right)$ plays the role of the frequency gain $\left|H_{k}(\omega)\right|^{2}, k=1,2$. Indeed, strict equality holds if one designs filters $H_{k}(\omega)$ 's such that their squared magnitudes equal $\cos \left(\omega \tau_{k}\right)$, which requires the use of 
fractional time-lags $|\tau| \leq 1 / 2$ such that $\cos \left(\omega \tau_{k}\right) \geq 0$ in the frequency band $[0, \pi]$. Another possibility is to set $\tau_{2}=0$ in Eq. (22) and then to note that the maximization of $\rho_{i}^{\mathrm{AMUSE}}$ is equivalent to that of $\rho_{i}^{\mathrm{AMUSE}}+1$. Thus,

$$
\rho_{i}^{\text {AMUSE }}+1=\frac{\int_{0}^{\pi}\left|Y_{i}(\omega)\right|^{2} \mid 1+\cos (\omega \tau) l d \omega}{\int_{0}^{\pi}\left|Y_{i}(\omega)\right|^{2} d \omega}, \quad \tau \neq 0
$$

which is identical to CP with $H_{1}(\omega)=\sqrt{1+\cos (\omega \tau)}$ and $H_{2}(\omega)=1$ for any time-lag $\tau$.

The above observations unveil an intimate relationship between AMUSE and CP. At the same time, it makes it clear that CP will suffer from all the same limitations as AMUSE when used in OMA, in particular the difficulty to identify complex mode shapes and to separate strongly coupled modes [10].

\subsection{Optimal pair of filters}

The previous discussions raise the question as whether there exists an optimal pair of filters to use in CP that maximizes the separation between modes.

A sensible objective is to maximize the contrast between eigenvalues $\rho_{i}, i=1, \ldots, n$. According to perturbation theory [16], this will strengthen the stability of the algorithm in the presence of estimation errors due to finite-sample size of the measurements and additive noise. Based on the results of subsection 3.1, a relevant measure of contrast between two adjacent modes $i=1,2$ is returned by the ratio of eigenvalues $\rho_{1}$ and $\rho_{2}$, which should be as large as possible. From Eq. (21), one finds

$$
\frac{\rho_{1}}{\rho_{2}} \approx \frac{\left|H_{1}\left(\omega_{1}\right)\right|^{2}\left|H_{2}\left(\omega_{2}\right)\right|^{2}}{\left|H_{2}\left(\omega_{1}\right)\right|^{2}\left|H_{1}\left(\omega_{2}\right)\right|^{2}} .
$$

The ratio is maximized by choosing transfer functions, $H_{1}(\omega)$ and $H_{2}(\omega)$, whose magnitudes are as different as possible at the resonance frequencies $\omega_{1}$ and $\omega_{2}$. This proves that the somewhat arbitrary filter weights initially suggested in [1,2,4] are by no means optimal for all purposes (nor are bandpass filters designed around the resonance frequencies of the structural modes as one could possibly think in a first place). Apart from maximizing the contrast between modes, the filters are so far not constrained to any specific shape (e.g. high-pass, band-pass, etc.), which leaves quite a flexibility in the method. Section 6 will further investigate this issue from an experimental point of view.

Noteworthy also is the antagonism between requests of having a strong contrast between filter gains at the resonance frequencies and the smoothness constraint imposed by condition (19), especially in the presence of closely spaced modes.

\section{A generalization of CP}

The interpretation of $\mathrm{CP}$ as in terms of invariance with respect to linear filters suggests immediate generalizations. A first one is to jointly diagonalize the cross-correlation matrices of several filtered versions of the signals instead of only two. This may be advantageous to prevent situations where some predefined filters $H_{1}(\omega)$ and $H_{2}(\omega)$ would be poorly adapted to separate a specific mixture of components (see Section 3.4). By multiplying the number of candidate filters an increased versatility of the algorithm is therefore expected as well as better numerical stability and robustness against estimation noise. A second generalization is to allow any type of filters with possibly complex coefficients, which will include SOBI as a particular case. This leads to a general algorithm which is formulated hereafter in the frequency domain.

Specifically, let us introduce a set of smooth complex frequency gains $\left\{G_{k}(\omega)\right\}_{k=0}^{K}, K \geq 2$. The objective is to find that separation matrix W which jointly diagonalizes the set of cross-correlation matrices $\left\langle\mathbf{x}^{(k)}(t) \mathbf{x}^{(k)}(t)^{T}\right\rangle$ for all $k=1, \ldots, K$ wherein $\mathbf{x}^{(k)}(t)$ stands for the system response filtered with transfer function $H_{k}(\omega)=\sqrt{G_{k}(\omega)}$. Using Parseval's identity, the cross-correlation matrices may be expressed as $\left\langle\mathbf{x}^{(k)}(t) \mathbf{x}^{(k)}(t)^{T}\right\rangle=\pi^{-1} \int_{0}^{\pi} \mathbf{x}(\omega) \mathbf{x}(\omega)^{H} G_{k}(\omega) d \omega$, where vector $\mathbf{x}(\omega)$ contains the Fourier transforms of the elements of vector $\mathbf{x}(t)$ and symbol ${ }^{H}$ stands for the conjugate transpose operator. Hence the following optimization problem

$$
\begin{aligned}
& \mathbf{W}=\operatorname{Arg} \min _{\mathbf{W}} \sum_{k=1}^{K}\left\|\operatorname{Off}\left(\mathbf{W}\left(\int_{0}^{\pi} \mathbf{S}_{x k}(\omega) G_{k}(\omega) d \omega\right) \mathbf{W}\right)\right\|^{2} \\
& \text { subjecttoW }\left(\int_{0}^{\pi} \mathbf{S}_{x x}(\omega) G_{0}(\omega) d \omega\right) \mathbf{W}^{T}=\mathbf{I}
\end{aligned}
$$

where Off stands for the operator which zeroes the diagonal elements of a matrix and $\|\cdots\|^{2}$ for the Frobenius norm of that matrix. A pseudo code to solve Eq. (25) is given in Appendix B.

Criterion (25) has several advantages.

- First it allows the inclusion of several different filters (not only two) with a greater chance that some of them will be optimally adapted to separating an unknown mixture of modes as explained in subsection 3.4.

- Second, the frequency domain formulation offers easy and flexible design of the filters as well as a superior control on the frequency bands of interest. Noteworthy is the fact that it applies equally well to stationary and transient responses, or mixtures of both. 
Table 1

Generalized CP and its particular cases.

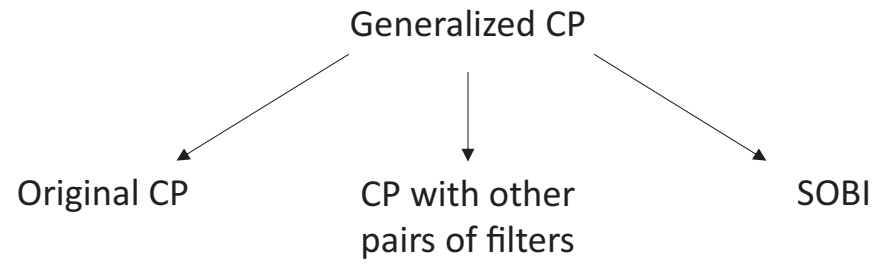

- Third, the proposed generalization accepts several other BSS algorithms as particular cases. The connection with AMUSE has already been established in subsection 3.3. The connection with SOBI - which is an extension of AMUSE seeking the diagonalization of cross-correlation matrices $\left\langle\mathbf{x}(t) \mathbf{x}\left(t-\tau_{i}\right)^{T}\right\rangle$ for several (more than two) time lags $\tau_{i}-$ is now immediate with $G_{k}(\omega)=\cos \left(\omega \tau_{i}\right)$ (see Eq. (22)) where negative values of $G_{k}(\omega)$ (and therefore complex values of $H_{k}(\omega)$ ) are allowed.

The fact the generalized CP includes other BSS algorithms as particular cases is theoretically interesting in an effort towards unification (see Table 1). It also necessarily places generalized CP at the same performance level of the BSS algorithms it embraces, in particular SOBI which, as mentioned in the introduction, probably stands as the one of the most famous algorithms used in OMA $[7,8,10,14]$.

\section{Numerical experiments}

The aim of this section is to compare the behavior of the original CP algorithm, as introduced in [1-4], with some of its generalizations addressed in the present paper as well as with the popular SOBI algorithm (a comprehensive analysis of SOBI in the field of modal analysis is given in [14], for instance).

The objective here is not to so much identify the best algorithm (multiple criteria would have to be defined for this purpose and tested on a much wider database), but rather to prove that all BSS algorithms falling in the generalization exposed in the present paper have more or less a similar behavior.

The numerical examples detailed in [10] are reproduced here in an effort to comply with reproducible research. In all experiments, three different versions of CP were implemented with constant parameters. The first one - hereafter denoted as CP1 follows the original derivation introduced in [1,4] and resumed by Eqs. (2) to (4). Values of $\lambda_{1}$ and $\lambda_{2}$ were set to $2^{-1 / 900000}$ and 0.5 , respectively, as advocated in [4]. This returned two transfer functions, $H_{1}(\omega)$ and $H_{2}(\omega)$, whose squared magnitudes are displayed in Fig. 2(a). The second CP algorithm - hereafter denoted as CP2 - was implemented by imposing the arbitrary transfer functions

$$
H_{1}(\omega)=1 \text { and } H_{2}(\omega)=\frac{1}{1-\frac{1}{2} e^{-j \omega}}
$$

displayed in Fig. 2(b), in order to demonstrate that the CP principle goes beyond the idea of predictability. The generalized CP algorithm - hereafter denoted as GCP - was designed according to the formulation of Section 4 with a set of 10 smooth FIR filters which are as different as possible, as shown in Fig. 2(c), in a hope to gain increased performance. The coefficients of the FIR filters are listed in Table 2. The three CP algorithms were systematically compared with SOBI set with 10 times-lags $\{\tau=0, \ldots, 9\}$.

\subsection{First experiment}

The first example is a 3 degree-of-freedom systems with mass, stiffness, and damping matrices given by

$$
\mathbf{M}=\left[\begin{array}{lll}
1 & 0 & 0 \\
0 & 2 & 0 \\
0 & 0 & 1
\end{array}\right], \quad \mathbf{K}=\left[\begin{array}{ccc}
5 & -1 & 0 \\
-1 & 4 & -3 \\
0 & -3 & 3.5
\end{array}\right], \quad \mathbf{C}=\frac{1}{21} \mathbf{M}+\frac{1}{120} \mathbf{K}=\left[\begin{array}{ccc}
0.0893 & -0.0083 & 0 \\
-0.0084 & 0.1286 & -0.0250 \\
0 & -0.0250 & 0.0768
\end{array}\right]
$$

The system has closely spaced modes with natural frequencies at $0.104 \mathrm{~Hz}, 0.342 \mathrm{~Hz}$, and $0.371 \mathrm{~Hz}$. The corresponding damping ratios are $4.0 \%, 2.0 \%$, and $2.0 \%$. The damping matrix was slightly modified as compared to [1] to make it exactly proportional in order to force real modes. Although the system has closely spaced modes, one can check that condition (20) is verified. The 3 degrees of freedom of the system were next driven with mutually uncorrelated white Gaussian noises in bandwidth [0;1 Hz] and the responses computed with numerical integration with sampling frequency $4 \mathrm{~Hz}$ for a total number of $2^{14}$ samples per channel.

The experiment was repeated with increasing values of additive white Gaussian noise with noise-to-signal ratios (NSR) of -30, $-20,-10$ and $0 \mathrm{~dB}$. In each case, 1000 runs (i.e. independent realizations of the random excitation) were performed and the results averaged together. 
(a)

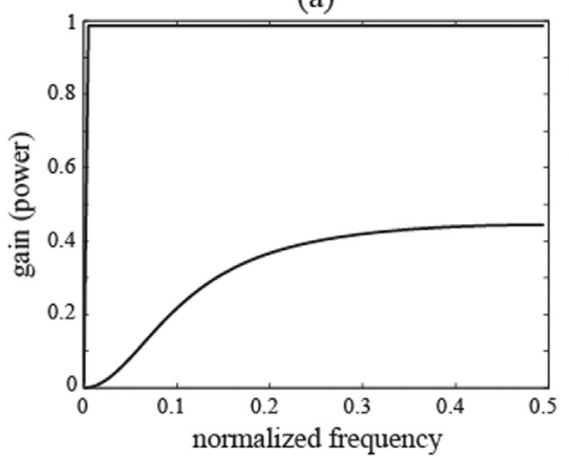

(b)

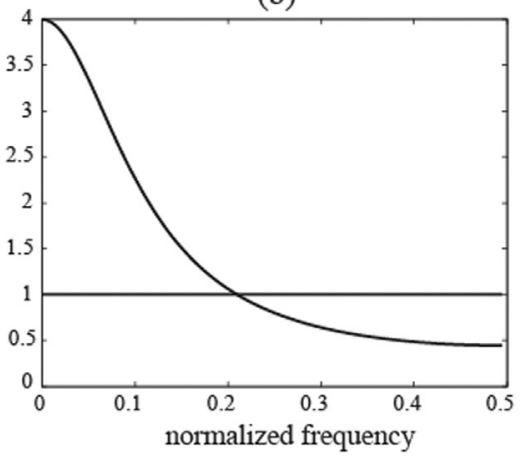

(c)

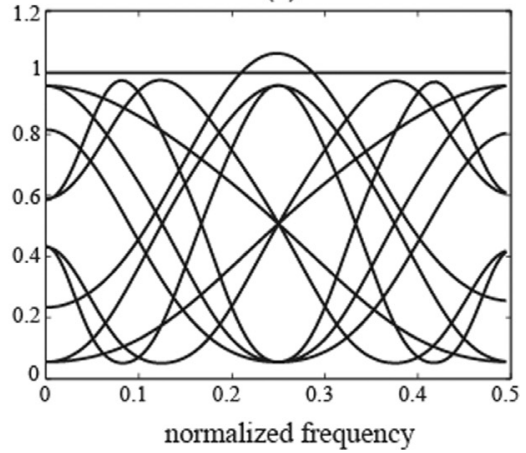

Fig. 2. Squared magnitudes of transfer functions $H_{1}(\omega)$ and $H_{2}(\omega)$ used in a) CP1, b) CP2, and c) GCP (frequency axis normalized by the sampling frequency).

Fig. 3 displays the power spectra of the separated modal contributions (averaged over 100 runs) for the three CP and SOBI algorithms in the noise-free case. Note that separated modes (identified by different colors) are returned in arbitrary order, as is always the case in BSS. Excellent separation is observed for all candidates, although very small cross-talks are noticeable in some of the separated spectra.

\subsection{Second experiment}

The same experiment was repeated after multiplying the damping matrix by a factor 10 . This produced strong damping ratios of $19.8 \%, 19.5 \%$, and $3.9 \%$. As a consequence, condition (20) was slightly violated by about a factor 3 . However, all algorithms could still achieve a reasonable and comparable separation, at least qualitatively as seen in Fig. 4. Overall, this demonstrates a certain robustness of $\mathrm{CP}$ to operate outside its theoretical range of applicability, when trying to separate heavily damped modes, just as it has been recognized for SOBI [14].

\subsection{Third experiment}

The third experiment has structural properties given as follows:

$$
\mathbf{M}=\left[\begin{array}{lll}
3 & 0 & 0 \\
0 & 2 & 0 \\
0 & 0 & 1
\end{array}\right], \quad \mathbf{K}=\left[\begin{array}{ccc}
4 & -2 & 0 \\
-2 & 4 & -2 \\
0 & -2 & 10
\end{array}\right], \quad \mathbf{C}=\left[\begin{array}{ccc}
0.1856 & -0.2290 & 0.9702 \\
-0.2290 & 0.0308 & -0.0297 \\
0.9702 & -0.0297 & 0.1241
\end{array}\right]
$$

which involves a non-proportional damping matrix not diagonalized by the normal modes. Thus, the difficulty will be to deal with complex mode shapes, a situation where the "plain-vanilla" version of SOBI has been shown to fall short [10]. The system has natural frequencies at $0.136 \mathrm{~Hz}, 0.247 \mathrm{~Hz}$, and $0.500 \mathrm{~Hz}$ and corresponding damping ratios of 3.0\%, $1.4 \%$, and $1.7 \%$.

Table 2

Coefficients of FIR filters used in GCP.

\begin{tabular}{|c|c|c|c|c|c|c|c|c|c|}
\hline H1 & $\mathrm{H} 2$ & H3 & $\mathrm{H} 4$ & H5 & H6 & $\mathrm{H} 7$ & H8 & H9 & H10 \\
\hline-0.00270176 & 0.00074839 & 0.00793082 & -0.00806997 & -0.00059523 & 0.00060147 & 0.00797567 & -0.00796622 & -0.00063695 & 0.00053202 \\
\hline-0.13447107 & 0.16656143 & -0.02631705 & -0.02635368 & -0.02692993 & 0.02682634 & $-4.3467 \mathrm{E}-05$ & -0.00011371 & -0.01751098 & 0.01753765 \\
\hline-0.00270004 & 0.0007479 & 0.17802799 & -0.17811503 & 0.02569651 & 0.02689128 & -0.02628817 & -0.02628395 & -0.00063534 & 0.00053071 \\
\hline 0.76262007 & 0.56666309 & 0.65950143 & 0.65964058 & -0.0927434 & 0.09263995 & $-4.3408 \mathrm{E}-05$ & -0.00011355 & -0.00076176 & 0.05332482 \\
\hline-0.00270004 & 0.0007479 & 0.17802799 & -0.17811503 & -0.0005937 & 0.00059992 & 0.17797072 & -0.17796129 & -0.00063414 & 0.00052973 \\
\hline-0.13447107 & 0.16656143 & -0.02631705 & -0.02635368 & 0.14946082 & -0.1495642 & $-4.3379 \mathrm{E}-05$ & -0.00011347 & -0.09831987 & 0.09837106 \\
\hline-0.00270176 & 0.00074839 & 0.00793082 & -0.00806997 & 0.65900912 & 0.66020236 & 0.65960524 & 0.65960945 & -0.00063335 & 0.00052908 \\
\hline 0 & 0 & 0 & 0 & 0.14946082 & -0.1495642 & $-4.3379 \mathrm{E}-05$ & -0.00011347 & 0.13490052 & -0.13494101 \\
\hline 0 & 0 & 0 & 0 & -0.0005937 & 0.00059992 & 0.17797072 & -0.17796129 & -0.00063295 & 0.00052875 \\
\hline 0 & 0 & 0 & 0 & -0.0927434 & 0.09263995 & $-4.3408 \mathrm{E}-05$ & -0.00011355 & 0.7342199 & 0.5848987 \\
\hline 0 & 0 & 0 & 0 & 0.02569651 & 0.02689128 & -0.02628817 & -0.02628395 & -0.00063295 & 0.00052875 \\
\hline 0 & 0 & 0 & 0 & -0.02692993 & 0.02682634 & $-4.3467 \mathrm{E}-05$ & -0.00011371 & 0.13490052 & -0.13494101 \\
\hline 0 & 0 & 0 & 0 & -0.00059523 & 0.00060147 & 0.00797567 & -0.00796622 & -0.00063335 & 0.00052908 \\
\hline 0 & 0 & 0 & 0 & 0 & 0 & 0 & 0 & -0.09831987 & 0.09837106 \\
\hline 0 & 0 & 0 & 0 & 0 & 0 & 0 & 0 & -0.00063414 & 0.00052973 \\
\hline 0 & 0 & 0 & 0 & 0 & 0 & 0 & 0 & -0.00076176 & 0.05332482 \\
\hline 0 & 0 & 0 & 0 & 0 & 0 & 0 & 0 & -0.00063534 & 0.00053071 \\
\hline 0 & 0 & 0 & 0 & 0 & 0 & 0 & 0 & -0.01751098 & 0.01753765 \\
\hline 0 & 0 & 0 & 0 & 0 & 0 & 0 & 0 & -0.00063695 & 0.00053202 \\
\hline
\end{tabular}


ARTICLE IN PRESS

J. Anton et al.

Mechanical Systems and Signal Processing 85 (2017) 773-788

(a)
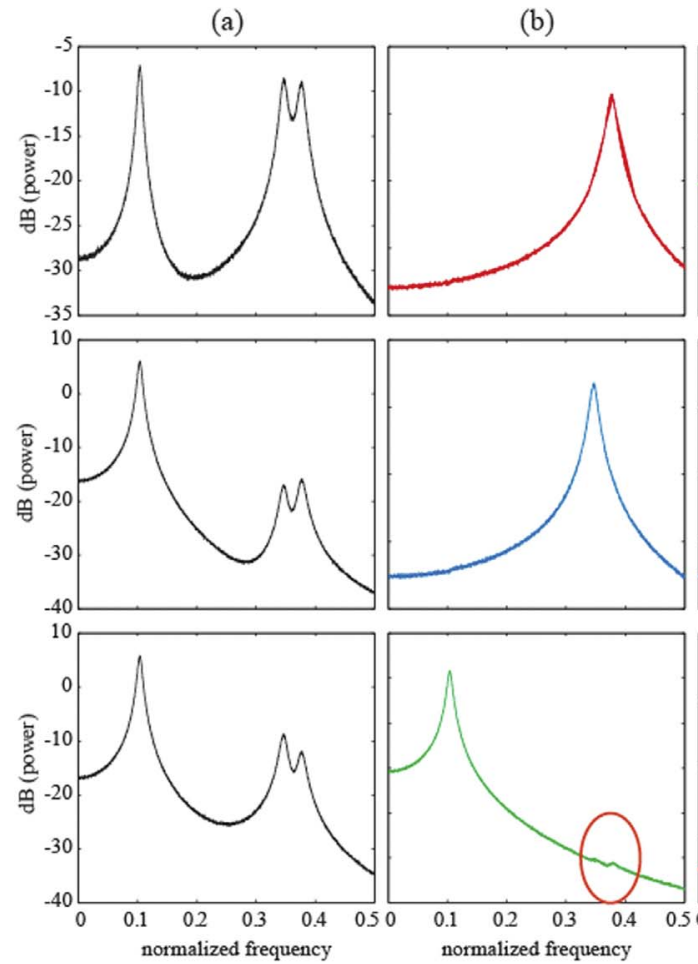

(c)

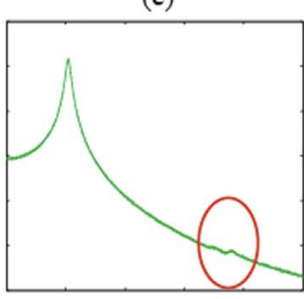

(d)
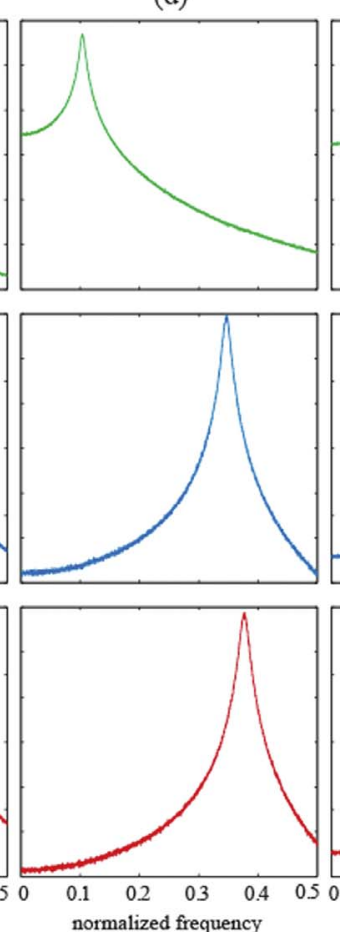

(e)
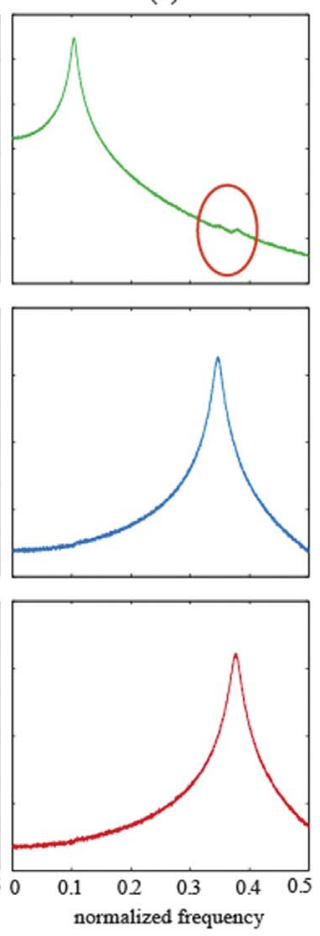

Fig. 3. Column (a): power spectra of the vibration responses. Columns (b) to (e): power spectra of the separated modal contributions with CP1, CP2, GCP, and SOBI, respectively. Light proportional damping. Red circles spot small residual cross-talks in the separated spectra. ( $\mathrm{dB}$ ref $1 \mathrm{~g}^{2} / \mathrm{Hz}$ ). (For interpretation of the references to color in this figure legend, the reader is referred to the web version of this article.)

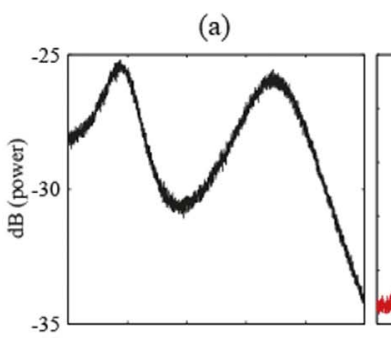

(b)

(c)

(d)

(e)
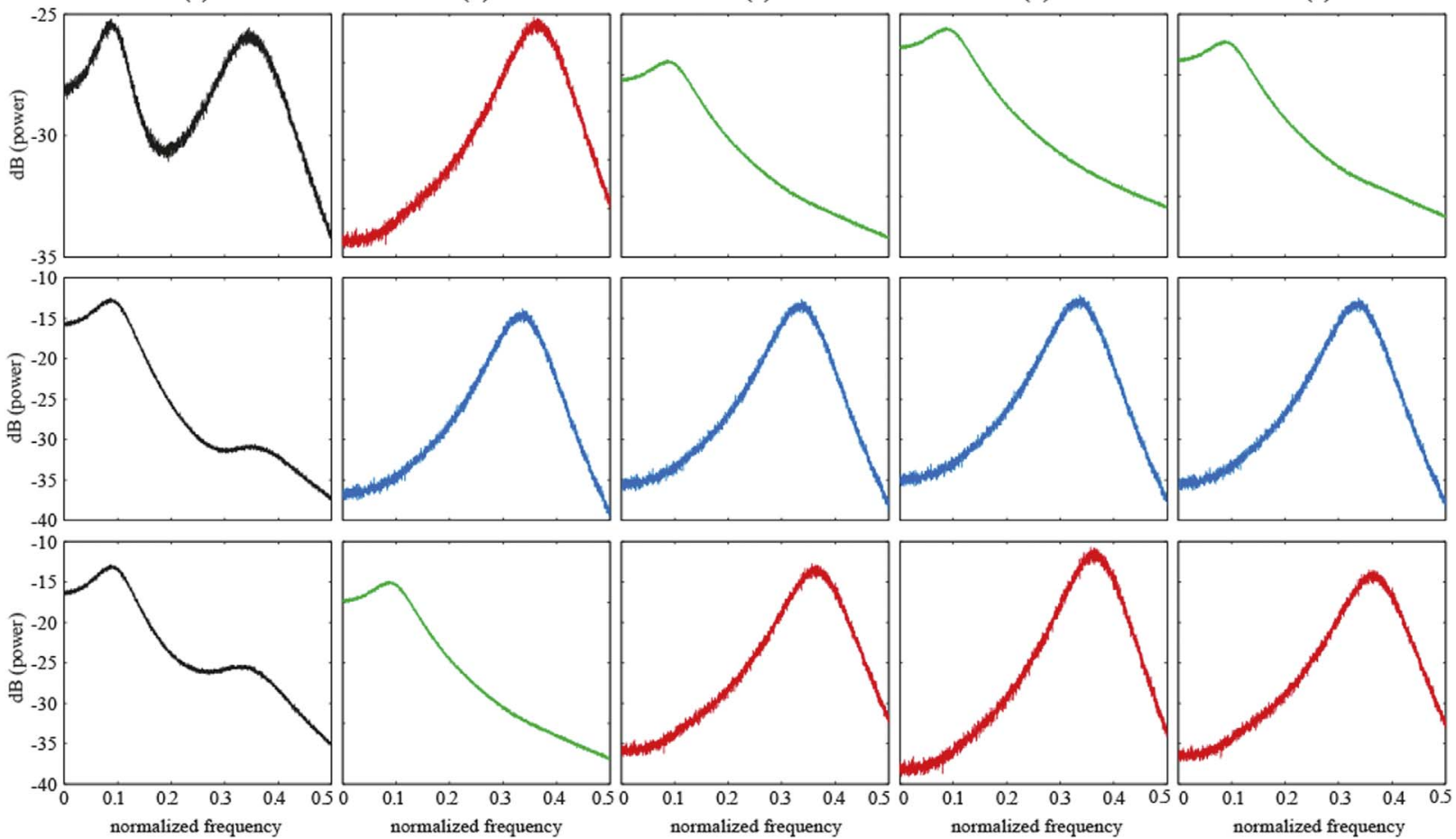

Fig. 4. Column (a): power spectra of the vibration responses. Columns (b) to (e): power spectra of the separated modal contributions with CP1, CP2, GCP, and SOBI, respectively. Strong proportional damping. ( $\mathrm{dB}$ ref $1 \mathrm{~g}^{2} / \mathrm{Hz}$ ).

781 
(a)
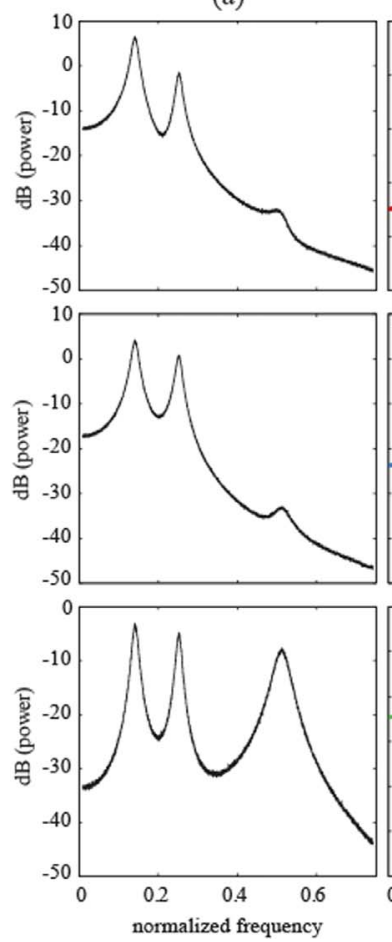

(b)
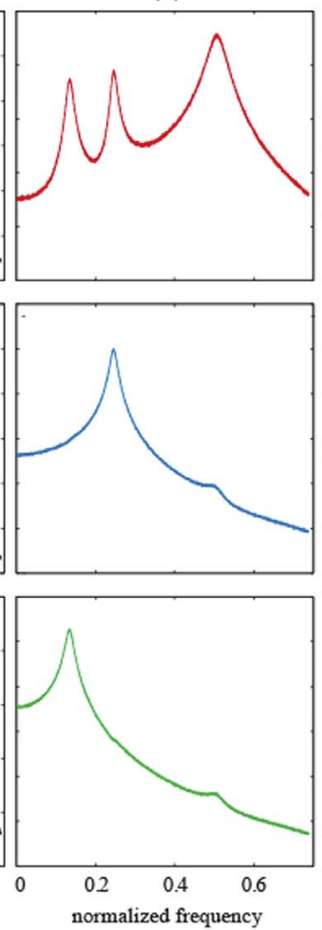

(c)
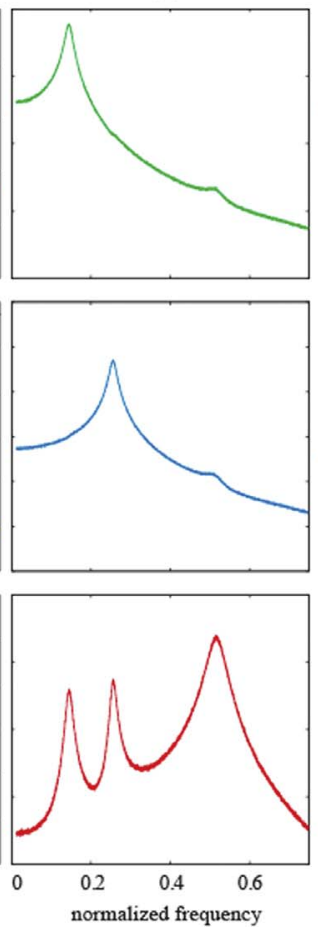

(d)
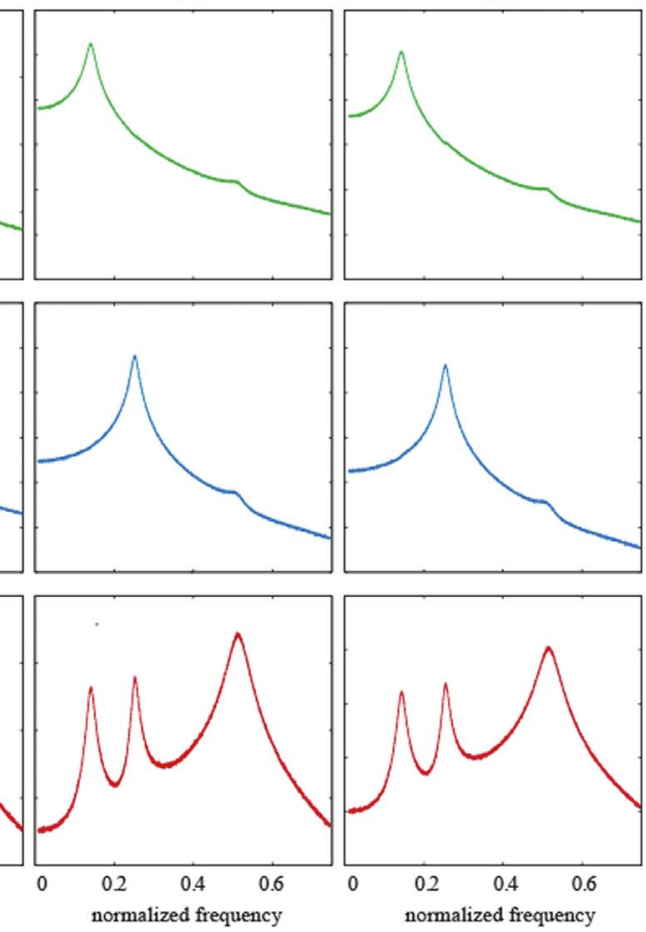

(e)
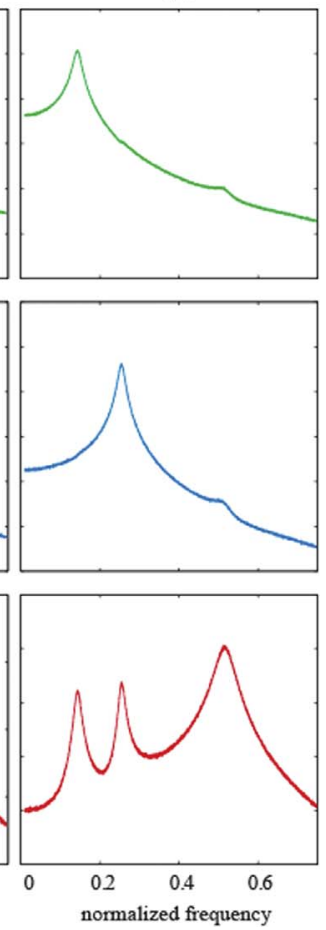

Fig. 5. Column (a): power spectra of the vibration responses. Columns (b) to (e): power spectra of the separated modal contributions with CP1, CP2, GCP, and SOBI, respectively. Non-proportional damping with complex mode shapes. (dB ref $1 \mathrm{~g}^{2} / \mathrm{Hz}$ ).

The separated components are displayed in Fig. 5. In agreement with the results of [10], SOBI is unable to separate the highest mode, yet the same phenomenon is observed for all three CP algorithms.

\subsection{Analysis of results}

In order to obtain a quantitative means of comparison of the separation results, the following figure of merit is proposed,

$$
e=\frac{\langle(\log S(\omega)-\langle\log S(\omega)\rangle)-(\log \hat{S}(\omega)-\langle\log \hat{S}(\omega)\rangle)\rangle}{\langle(\log \hat{S}(\omega)-\langle\log \hat{S}(\omega)\rangle)\rangle},
$$

which measures the relative logarithmic error between the actual spectra $S(\omega)$ and the separated ones, $\hat{S}(\omega)$, wherein $\langle\cdots\rangle=\int_{0}^{\pi}(\cdots) d \omega$ denotes frequency averaging (note that the subtraction of the logarithm average is to correct for the unknown scale of the separated modal contributions). The relative error $e$ is averaged over 1000 independent runs. This figure of merit should reflect very well the capacity of correctly identifying the natural frequencies and damping ratios of the structural modes in a subsequent step following BSS. It is displayed in Fig. 6 for the three experiments reported in the previous subsections.

The first experiment with light proportional damping clearly returns very good separation results for all noise-to-signal-ratios (NSR) less or equal to $-10 \mathrm{~dB}$ and this independently of the BSS algorithm (Fig. 6(a)). The CP1 and the CP2 curves are superimposed and show a relative error slightly superior to SOBI and GCP - also superimposed - for NSR $\leq-10 \mathrm{~dB}$. Relative errors for $\mathrm{NSR}=0 \mathrm{~dB}$ are quite high and therefore comparisons of performance are difficult in this range.

In the second experiment with strong proportional damping, GCP has the smallest relative error for NSR $\leq-10 \mathrm{~dB}$ and CP1 and CP2 (again superimposed) have the highest (Fig. 6(b)). Although separation was still successful (see Fig. 4), the price to pay is a notable reduction in the figure of merits. Again the high values of the relative errors for NSR $=0 \mathrm{~dB}$ are not amenable to meaningful comparisons.

The figures of merits of non-proportional damping with complex mode shapes are displayed in Fig. 6(c), where it is seen that all algorithms fail equally in this difficult situation. This illustrates an important limit of the approach. This result seems in disagreement with a conclusion of [1] where CP was claimed to be able to separate complex modes. It is believed that [1] applied CP on the analytical signal and that the capability of separating complex modes then came from this pre-processing (as demonstrated in [10]) rather than from the method itself.

In conclusion of this section, it is observed that: 
(a)

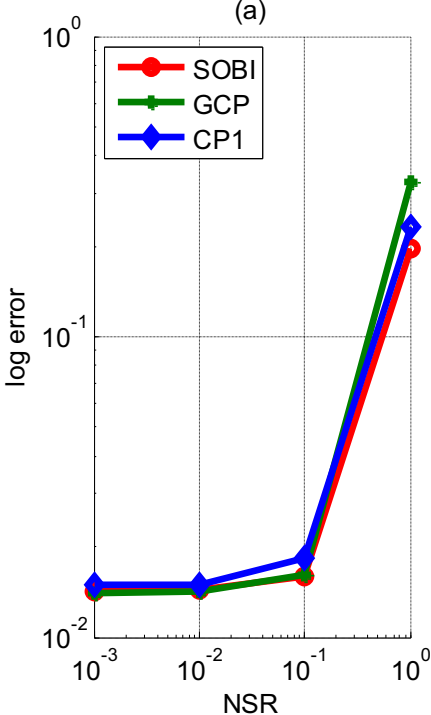

(b)

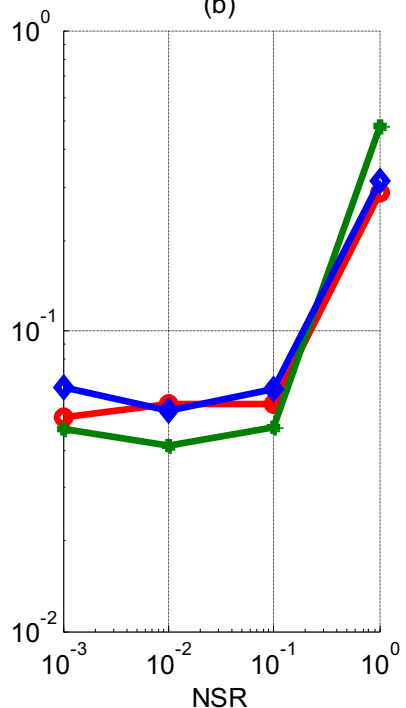

(c)

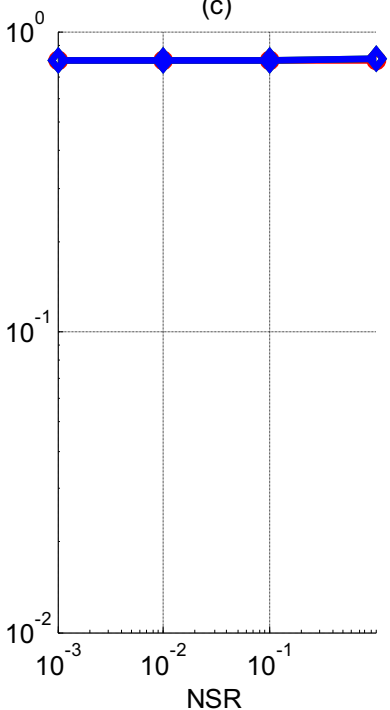

Fig. 6. Relative logarithmic error (dimensionless) as a function of noise-to-signal-ratio (dimensionless): a) light proportional damping (first experiment), b) strong proportional damping (second experiment), c) non-proportional damping with complex mode shapes (third experiment).

- CP1 and CP2 (with the specific choice of filters given in this section) perform identically, thus demonstrating that the CP principle applies with any smooth filter.

- $\mathrm{CP} 1$ and CP2 both evidence poorer performance than SOBI in these examples.

- GCP is able to steer the performance of CP to the level of SOBI and even higher, thus demonstrating the advantage of using more than 2 filters.

\section{Experimental investigation of the optimality filters}

Another experimental issue to investigate is the choice of the "best" set of filters for the application of CP in its generalized versions. Previous tests were conducted by using arbitrary FIR filters, yet as shown in Section 3.4 the method can surely be optimized by designing filters that are more adapted to the modal characteristics of a system. The corresponding frequency gains should be reasonably smooth, nearly constant across the mode bandwidth, and at the same time as contrasted as possible (see Section 3).

This is first illustrated for the separation of two modes by means of two filters (method CP2). As discussed in subsection 3.4, for an optimal separation the two filters should return frequency gains as different as possible, each one highlighting a particular mode of the system. This is illustrated in Figs. 7 and 8 where a pair of high-pass filters has been used to test the spectral separation of adjacent modes. In order to get an efficient separation, not only do the filters have to cut off the modes, but they should also magnify them in a different manner. As seen in Fig. 9, the best results are obtained when the slope of the high-pass filters crosses a mode. On the contrary, Figs. 7 and 8 illustrates two situations where the pair of filters locally put the same relative weights on the two modes (i. e. the ratio $\rho_{1} / \rho_{2}$ in Eq. (24) is equal to its minimal value of one), which is clearly prone to failure.

Next the same experiment is repeated by using the generalized formulation of CP and spanning the frequency band of interest with a bank of 8 high-pass filters (see Fig. 10). By construction, there is a better chance that some of the filters in the set will be close to optimal. The good performance of the separation can be clearly seen in Fig. 10, together with a comparison with same-order SOBI which fails in the identification of the two closely spaced modes.

Other interesting sets of filters which have been tested are octave bands and fractional octave bands. The rational beyond this approach is that each filter will enhance a specific (narrow) band of frequencies and therefore magnify a prominent mode present in that band (if any) while partially attenuating the "out-of-band" modes. The approach is illustrated in Fig. 11 where separation is achieved correctly with GCP with 8 filters and not with the same-order SOBI.

\section{Application to experimental data}

The methodology is finally validated by analysis of experimental data. These come from a scale 5-story building located in the Department of Mechanical and Aerospace Engineering at Politecnico di Torino. The investigated structure, whose scheme is shown in Fig. 12, is composed of five aluminum decks linked by thin steel beams; it might be reasonably considered as a 5-DOF system as the plate stiffness is much higher than the flexural stiffness of the vertical beams. Its transient response is measured and analyzed with the generalized version of CP by using sets of sinusoidal (GCP-S), octave-band (GCP-O) and high-pass (GCP$\mathrm{H}$ ) filters in comparison to SOBI. Sampling frequency is normalized at $1 \mathrm{~Hz}$ and modes have been searched in the normalized 

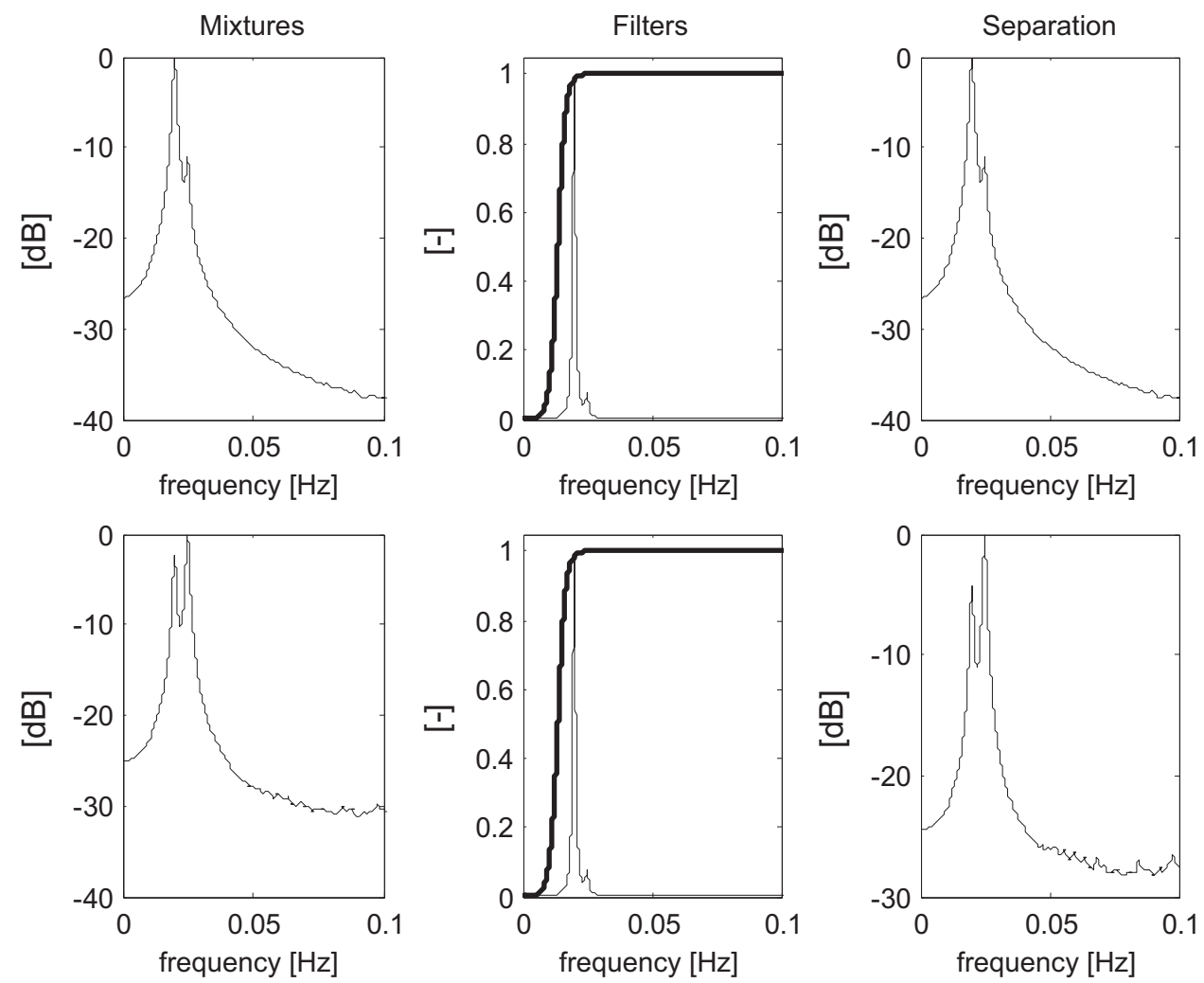

Fig. 7. Application of $\mathrm{CP}$ by employing a pair of identical filters (dB ref $1 \mathrm{~g}^{2} / \mathrm{Hz}$ ).
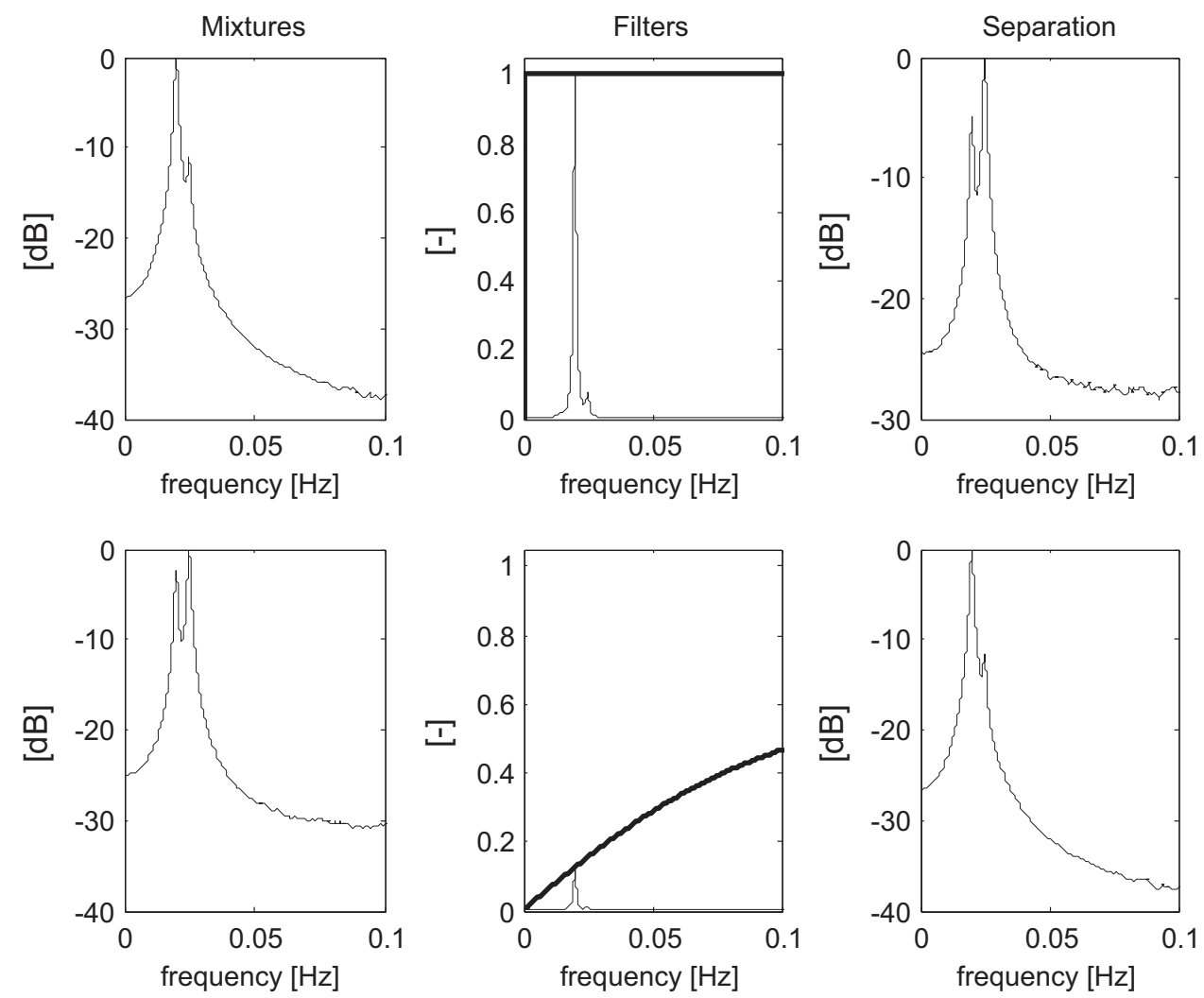

Fig. 8. Application of $\mathrm{CP}$ by employing a filter outside the frequency band of interest ( $\mathrm{dB}$ ref $1 \mathrm{~g}^{2} / \mathrm{Hz}$ ). 

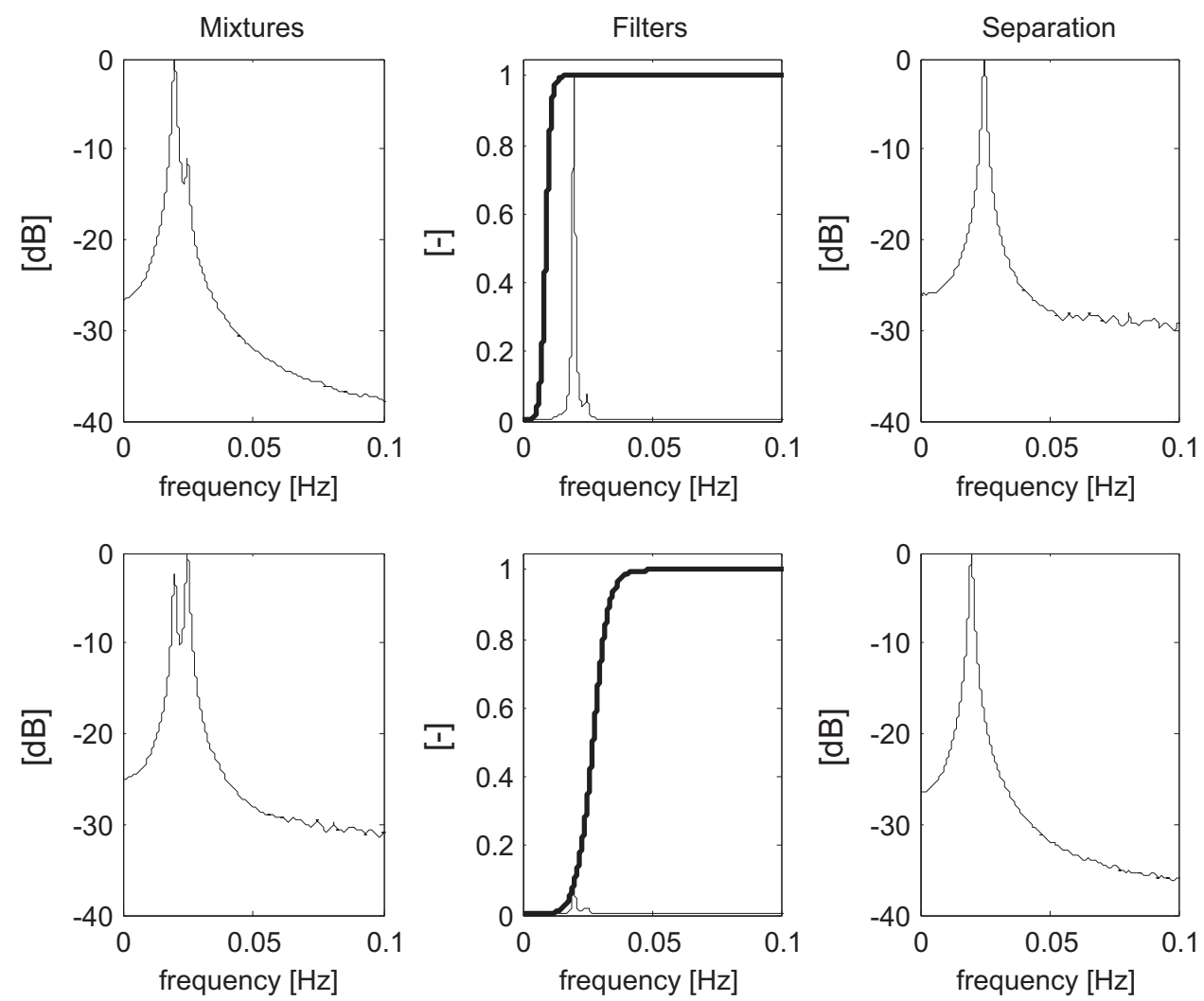

Fig. 9. Application of CP by employing a "good" pair of filters (dB ref $1 \mathrm{~g}^{2} / \mathrm{Hz}$ ).
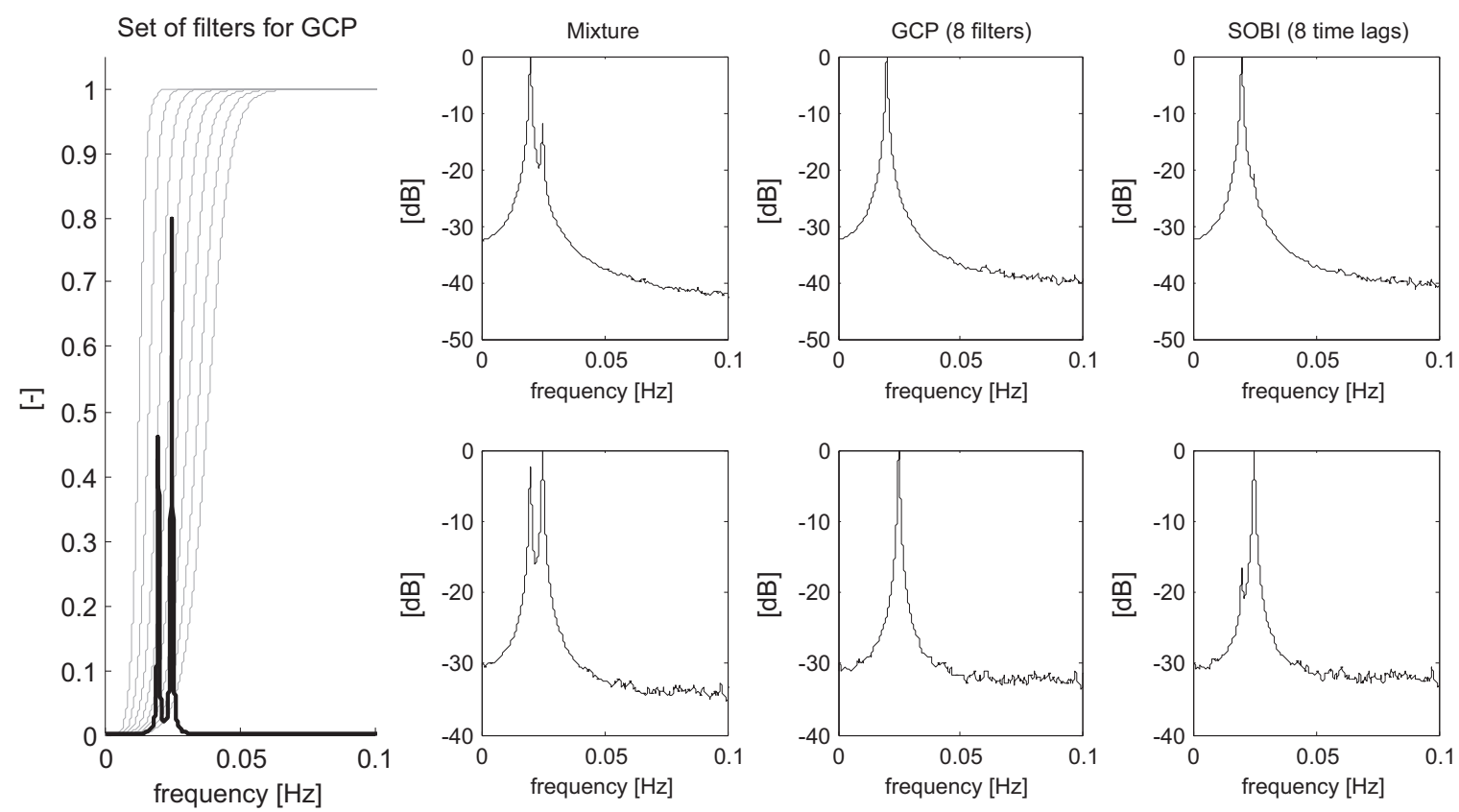

Fig. 10. Generalized CP with a set of high-pass filters and comparison with SOBI (dB ref $1 \mathrm{~g}^{2} / \mathrm{Hz}$ ).

frequency interval [ $\left.\begin{array}{ll}0 & 0.3\end{array}\right] \mathrm{Hz}$. The same order (number of filters for GCP and number of time lags for SOBI) is used for all techniques and it is set to 10 . 

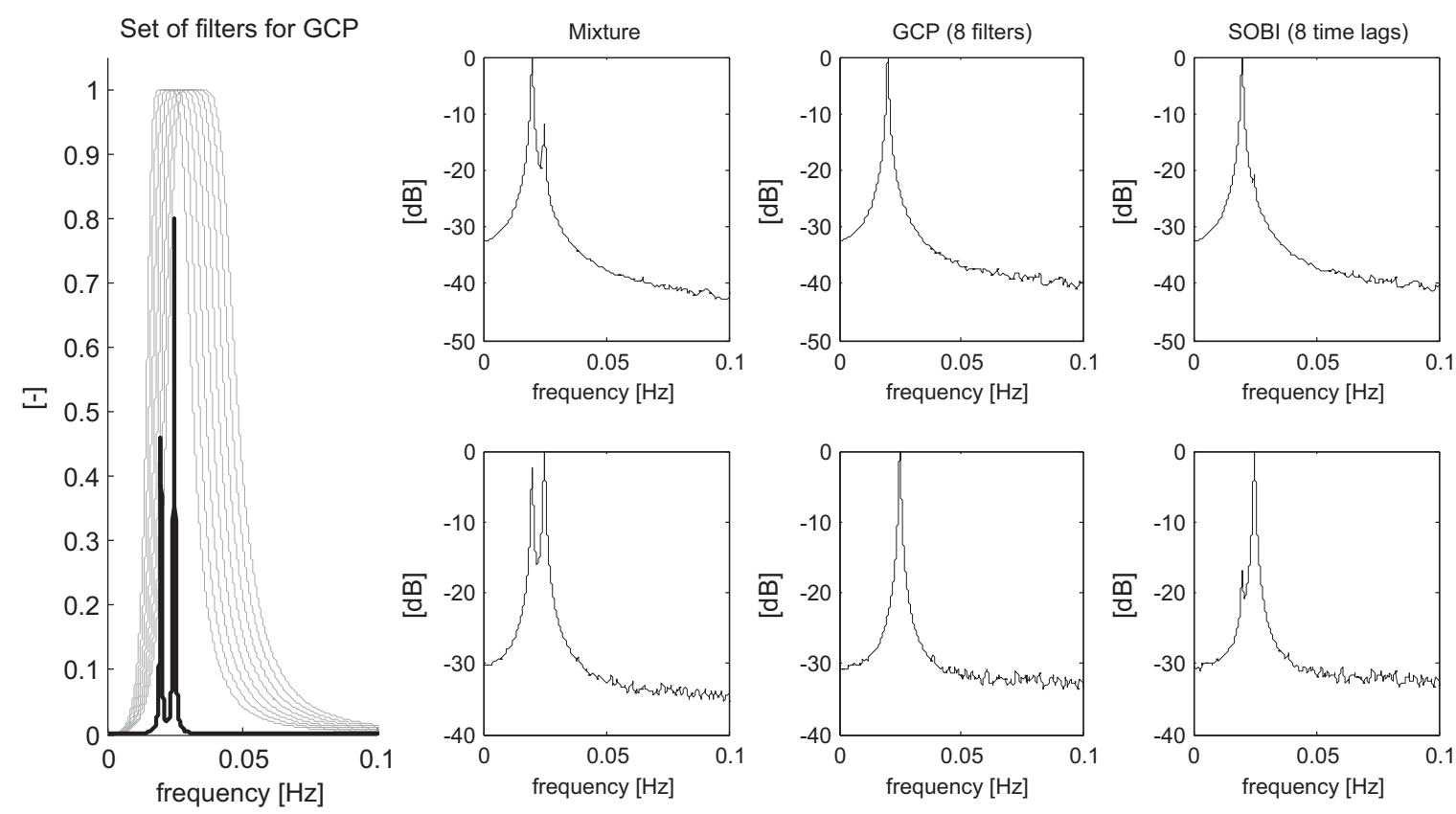

Fig. 11. Generalized CP with a set of third-octave band filters and comparison with SOBI (dB ref $1 \mathrm{~g}^{2} / \mathrm{Hz}$ ).

The modes separated by GCP using the different sets of filters and by SOBI are compared in Fig. 13. The four algorithms were able to separate the modes of the structure, in particular the two pairs of adjacent modes at $0.022-0.024 \mathrm{~Hz}$ and $0.077-0.080 \mathrm{~Hz}$. SOBI proved slightly inferior in the identification of the mode at $0.101 \mathrm{~Hz}$ (some small cross-talks are noticeable on the fourth separated spectrum).

\section{Conclusion}

This aim of this paper was to provide a theoretical analysis of the CP technique recently introduced in [1-3] within the context of operational modal analysis. Since CP is claimed to blindly separate the individual modal contributions of a system from its vibration responses only, its potential interest to practical applications is not to be underestimated. The main result of the paper is that $\mathrm{CP}$, in its original version, is not as versatile as it first appeared. It has been proved that the only modal contributions that it can separate exactly (without resorting to the assumption of statistical independence) are made of pure sines: these are the responses of purely conservative systems. The good news, however, is that CP is quite robust against this condition and that it works remarkably well in separating damped modes, as long as the corresponding modal coordinates are slowly modulated sinusoids. This is actually reminiscent to other BSS algorithms when applied to modal analysis. Robustness against additive noise has also been investigated. Another result of the paper proves that $\mathrm{CP}$ can be generalized to the use of any smooth filters and not just those initially designed in [4]. This conveys the technique a more general interpretation than it was initially given in terms of temporal predictability; namely, CP tries to separate components which are as least dispersive (i.e. invariant under linear filtering) as possible. This indicates that "complexity" is actually to be measured by a propensity of dispersion rather than by predictability. The same result also suggests a generalization of CP which, in a similar spirit to SOBI, jointly diagonalizes the cross-correlation matrices of an arbitrary number of filtered versions of the signals. Numerical simulations have shown that generalized CP is likely to improve the performances of the plain-vanilla CP, similarly to SOBI which actually happens to be a particular case of the proposed generalization. Other improvements are likely to result from the findings of this paper. One perspective is to use the first separation returned by a general purpose family of filters and then iterate to design filters better optimized to the identified modes.

\section{Acknowledgments}

This work was performed within the framework of the LabExCeLyA ("Center Lyonnais d'Acoustique", ANR-10-LABX-60).

\section{Appendix A. : proof of Eq. (6)}

Since $Y_{i}(\omega)$ is complex valued, the perturbation is sought by keeping $Y_{i}(\omega)^{*}$ constant. Therefore, 


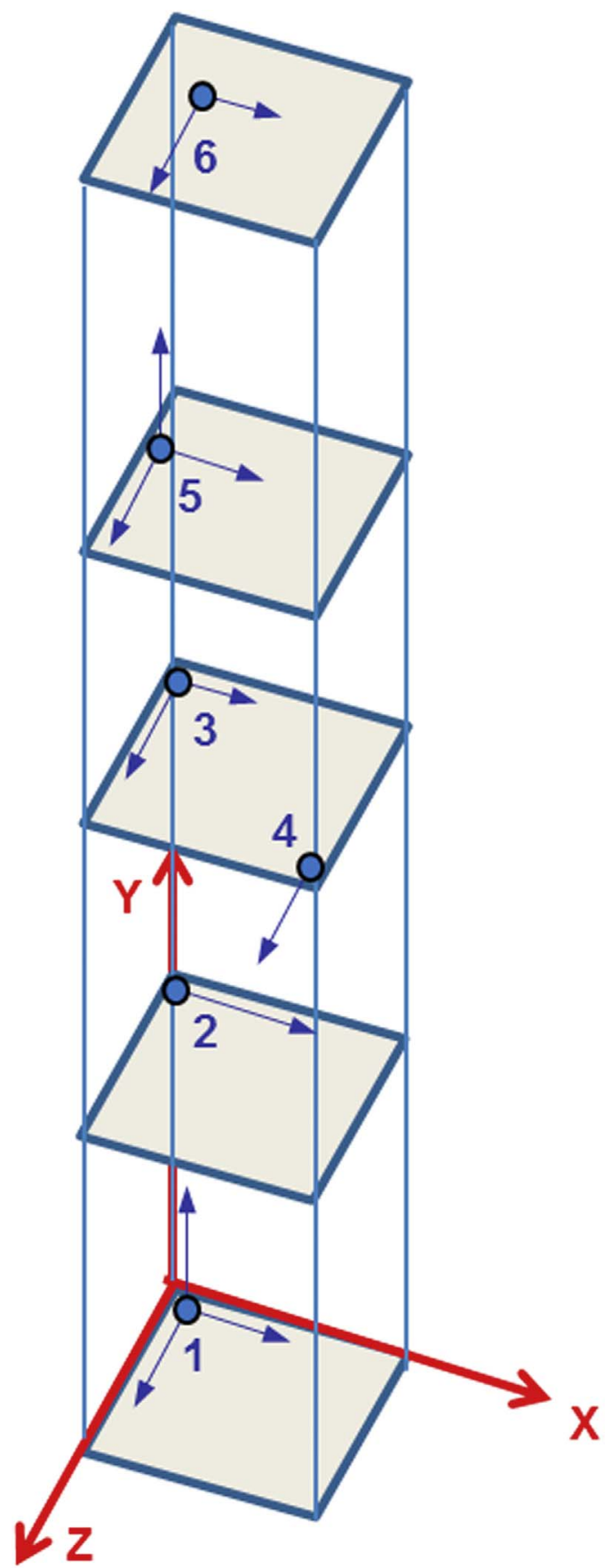

Fig. 12. Scheme of the structure used in the experiment.

$$
\rho_{i}\left(Y_{i}(\omega)\right)=\rho_{i}\left(Y_{i}^{0}(\omega)+\delta Y_{i}(\omega)\right)=\frac{\int_{0}^{\pi}\left|H_{1}(\omega)\left(Y_{i}^{0}(\omega)+\delta Y_{i}(\omega)\right)\right|^{2} d \omega}{\int_{0}^{\pi}\left|H_{2}(\omega)\left(Y_{i}^{0}(\omega)+\delta Y_{i}(\omega)\right)\right|^{2} d \omega} .
$$

Keeping only the first-order terms in the Taylor expansion,

$$
\begin{aligned}
\rho_{i}\left(Y_{i}(\omega)\right) & \approx \frac{U+\int_{0}^{z}\left|H_{1}(\omega)\right|^{2} \delta Y_{i}(\omega) Y_{i}^{\omega}(\omega)^{*} d \omega}{V+\int_{0}^{\pi}\left|H_{2}(\omega)\right|^{2} \delta Y_{i}(\omega) Y_{i}^{\nu}(\omega)^{*} d \omega} \approx \frac{U+\int_{0}^{\pi}\left|H_{1}(\omega)\right|^{2} \delta Y_{i}(\omega) Y_{l}^{\phi}(\omega)^{*} d \omega-\frac{U}{V} \int_{0}^{z}\left|H_{2}(\omega)\right|^{2} \delta Y_{i}(\omega) Y_{i}^{\omega}(\omega)^{*} d \omega}{V} \\
& =\frac{U}{V}+\frac{1}{V} \int_{0}^{\pi}\left|H_{1}(\omega)\right|^{2} \delta Y_{i}(\omega) Y_{i}^{\omega}(\omega)^{*} d \omega-\frac{U}{V^{2}} \int_{0}^{\pi}\left|H_{2}(\omega)\right|^{2} \delta Y_{i}(\omega) Y_{i}^{\omega}(\omega)^{*} d \omega,
\end{aligned}
$$



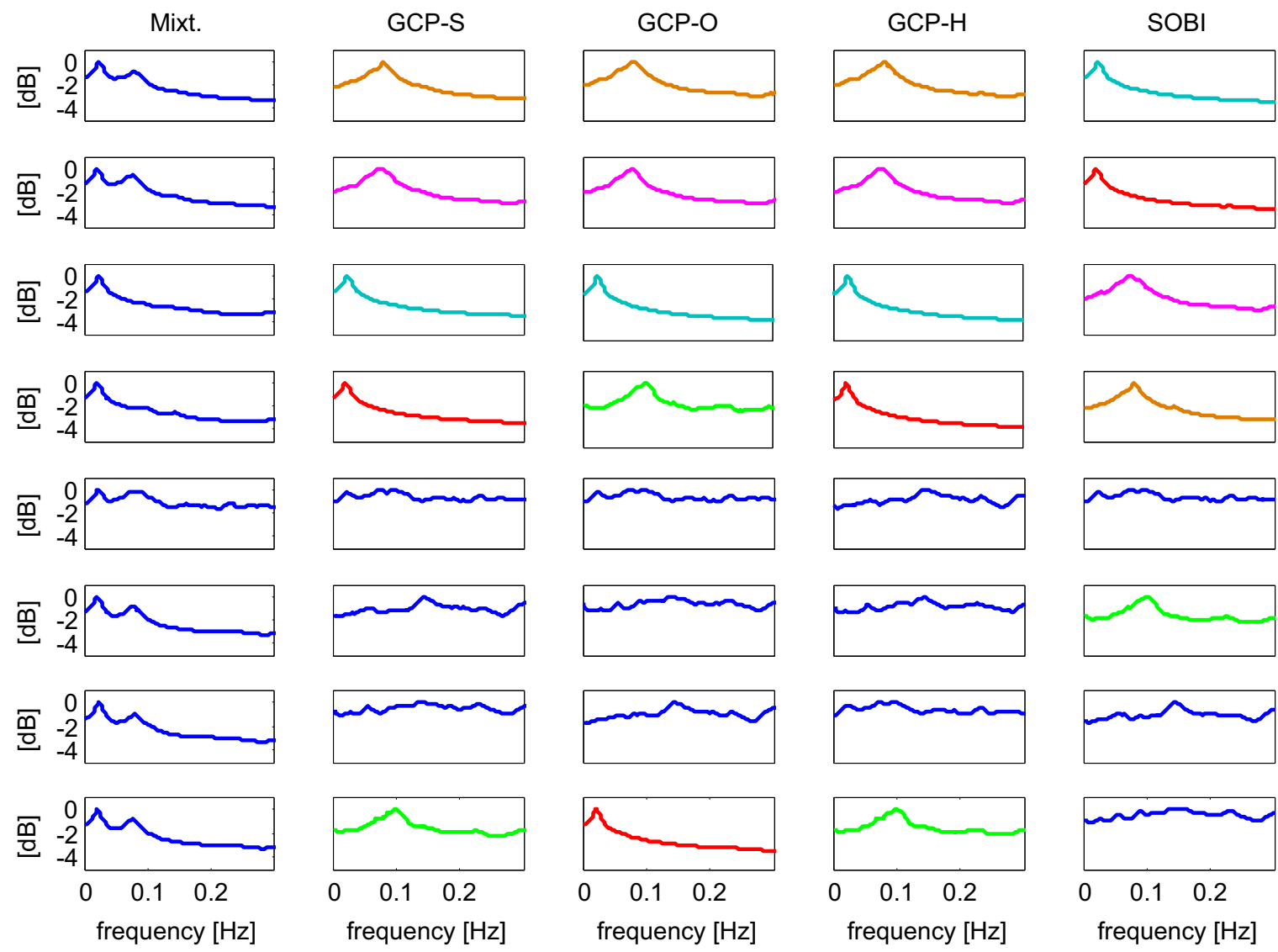

Fig. 13. Comparison of different (but same-order) versions of GCP applied on experimental data: sinusoidal filters (GCP-S), octave-band filters (GCP-O) high-pass filters (GCP-H) and SOBI (dB ref $\left.1 \mathrm{~g}^{2} / \mathrm{Hz}\right)$.

Eq. (6) immediately follows after subtracting $\rho_{i}\left(Y_{i}^{0}(\omega)\right)=U / V$.

Appendix B. : pseudo code to solve Eq. (25)

Step 1: Compute the set of matrices $\mathbf{S}_{k}=\int_{0}^{\pi} \mathbf{S}_{x x}(\omega) G_{k}(\omega) d \omega, k=0, \ldots, K$.

Step 2: Compute the eigenvalue decomposition $\mathbf{S}_{0}=\mathbf{U} \boldsymbol{\Lambda} \mathbf{U}^{H}$ of matrix $\mathbf{S}_{0}$, where $\boldsymbol{\Lambda}$ and $\mathbf{U}$ are diagonal and unitary matrices, respectively.

Step 3: Compute the matrices

$$
\hat{\mathbf{S}}_{k}=\mathbf{U}^{H} \boldsymbol{\Lambda}^{-\frac{1}{2}} \mathbf{S}_{k} \boldsymbol{\Lambda}^{-\frac{1}{2}} \mathbf{U}, \quad k=1, \ldots, K .
$$

Step 4: Find $\mathbf{W}$ which jointly diagonalizes the set of matrices $\left\{\tilde{\mathbf{S}}_{k}\right\}, k=1, \ldots, K$.

Any joint approximate diagonalization solver ${ }^{2}$ can be used in step 4 [20]; solvers dedicated to positive definite matrices [21] will require $G_{k}(\omega) \geq 0$ in Eq. (25).

\section{References}

[1] Y. Yang, S. Nagarajaiah, Blind modal identification of output-only structures in time-domain based on complexity pursuit, Earthq. Eng. Struct. Dyn. 42 (13) (2013) 1885-1905.

[2] Y. Yang, S. Nagarajaiah, S. Output-only, Modal identification by compressed sensing: non-uniform low-rate random sampling, Mech. Syst. Signal Process. 5657 (2015) 15-34.

[3] Y. Yang, S. Nagarajaiah, Structural damage identification via a combination of blind feature extraction and sparse representation classification, Mech. Syst. Signal Process. 45 (2014) 1-23.

[4] J.V. Stone, Blind source separation using temporal predictability, Neural Comput. 13 (2001) 1559-1574.

[5] J. Antoni, Blind separation of vibration components: principle and demonstrations, Mech. Syst. Signal Process. 19 (2005) 1166-1180.

[6] G. Kerschen, F. Poncelet, J.C. Golinval, Physical interpretation of independent component analysis in structural dynamics, Mech. Syst. Signal Process. 21 (2007)

\footnotetext{
${ }^{2}$ A Matlab ${ }^{\circledast}$ code that implements GCP can be downloaded at http://www.mathworks.com/matlabcentral/fileexchange/
} 
$1561-1575$.

[7] F. Poncelet, G. Kerschen, J.C. Golinval, D. Verhelst, Output-only modal analysis using blind source separation techniques, Mech. Syst. Signal Process. 21 (2007) $2335-2358$.

[8] S. Chauhan, R. Martell, R.J. Allemang, D. L. Brown, Application of Independent Component Analysis and Blind Source Separation Techniques to Operational Modal Analysis, in: Proceedings of the 25th IMAC, Orlando (FL), USA (2007).

[9] W. Zhou, D. Chelidze, Blind source separation based vibration mode identification, Mech. Syst. Signal Process. 21 (8) (2007) $3072-3087$.

[10] S.I. McNeill, D.C. Zimmerman, A framework for blind identification using joint approximate diagonalisation, Mech. Syst. Signal Process. 22 (2008) 1526-1548.

[11] B. Hazra, S. Narasimhan, Wavelet-based blind identification of the ucla factor building using ambient and earthquake responses, Smart Mater. Struct. 19 (2) (2010) 025005

[12] V.H. Nguyen, J.-C. Golinval, Damage detection using blind source separation techniques, 29th IMAC, in: Proceedings of A Conference on Structural Dynamics, (2011)(Jacksonville, FL, USA) pp.45-56.

[13] M. Spiridonakos, N. Yadav, and E. Chatzi,Identification and Damage Detection of a Shear Frame Model based on a Blind Source Separation method, in: Proceedings of the 7th Workshop on Structural Health Monitoring, Nantes, France, July 8-11 2014.

[14] J. Antoni, S. Chauhan, A study and extension of second-order blind source separation to operational modal analysis, J. Sound Vib. 332 (4) (2013) 1079-1106.

[15] A. Hyvärinen, Complexity pursuit: separating interesting components from time series, Neural Comput. 13 (2001) 883-898.

[16] J. Franklin, Matrix Theory, Dover, New York, 2000.

[17] A. Belouchrani, K.K. Abed-Meraim, J.F. Cardoso, E. Moulines, Second order blind separation of correlated sources, in: Proceedings of the International Conference on Digital Signal Processing, 1993, pp. 346-35.

[18] L. Tong, V.C. Soon. Y. Huang, R. Liu, AMUSE: a new blind identification algorithm (New Orleans, LA) in:Proceedings of the IEEE ISCAS 3, 1990, pp.17841787.

[19] J. Antoni, S. Chauhan, M. Monnier, K. Gryllias, Least action criteria for blind separation of structural modes, Mech. Ind. 14 (6) (2014) $397-411$.

[20] S. Degerine, E. Kane, A Comparative Study of Approximate Joint Diagonalization Algorithms for Blind Source Separation in Presence of Additive Noise, IEEE Trans. Signal Process. 55 (6) (2007) 3022-3031.

[21] D.T. Pham, Joint Approximate Diagonalization of Positive Definite Hermitian Matrices, SIAM. J. Matrix Anal. Appl., vol. 22 (4), pp.1136-1152. 